\title{
LIMITING ABSORPTION PRINCIPLE, GENERALIZED EIGENFUNCTIONS AND SCATTERING MATRIX FOR LAPLACE OPERATORS WITH BOUNDARY CONDITIONS ON HYPERSURFACES
}

\author{
ANDREA MANTILE, ANDREA POSILICANO, AND MOURAD SINI
}

\begin{abstract}
We provide a limiting absorption principle for the selfadjoint realizations of Laplace operators corresponding to boundary conditions on (relatively open parts $\Sigma$ of) compact hypersurfaces $\Gamma=$ $\partial \Omega, \Omega \subset \mathbb{R}^{n}$. For any of such self-adjoint operators we also provide the generalized eigenfunctions and the scattering matrix; both these objects are written in terms of operator-valued Weyl functions. We make use of a Kreйn-type formula which provides the resolvent difference between the operator corresponding to self-adjoint boundary conditions on the hypersurface and the free Laplacian on the whole space $\mathbb{R}^{n}$. Our results apply to all standard examples of boundary conditions, like Dirichlet, Neumann, Robin, $\delta$ and $\delta^{\prime}$-type, either assigned on $\Gamma$ or on $\Sigma \subset \Gamma$.
\end{abstract}

\section{INTRODUCTION}

Given an open bounded set $\Omega \subset \mathbb{R}^{n}$ with smooth boundary $\Gamma$, let $\Delta^{\circ}$ denote the not positive symmetric operator in $L^{2}\left(\mathbb{R}^{n}\right)$ given by the restriction of the Laplacian to $C_{\text {comp }}^{\infty}\left(\mathbb{R}^{n} \backslash \Gamma\right)$. In the recent paper [29], we provided the complete family of self-adjoint extensions of $\Delta^{\circ}$ and a Kreln-type formula giving the resolvent difference between any extension and the self-adjoint (free) Laplacian $\Delta$ with domain $H^{2}\left(\mathbb{R}^{n}\right.$ ) (we recall these results in Theorem 3.1). Some sub-families of extensions have been considered in [16] and [22] by a quadratic form approach and in [9] by quasi boundary triple theory. In particular, in [9, Section 4], Schatten-von Neumann estimates for the difference of the powers of the resolvent of the free and self-adjoint extensions corresponding to $\delta$-type boundary conditions (supported either on $\Gamma$ or on $\Sigma \subset \Gamma$ ) and $\delta^{\prime}$-type ones (supported on $\Gamma$ ) are provided; these give existence and completeness of the wave operators of the associated scattering systems. In [29, Theorems 4.10 and 4.11] we extended such kind of Schatten-von Neumann estimates to a larger class containing, for example, self-adjoint extensions corresponding to Dirichlet, Neumann, Robin, $\delta$ and $\delta^{\prime}$-type conditions, either assigned on $\Gamma$ or on $\Sigma \subset \Gamma$, where $\Sigma$ is relatively open with a Lipschitz boundary. To this concern we recall that estimates 
for the difference of the powers of the resolvents and their applications to scattering in exterior domains first appeared in the pioneering work by Birman [14.

Let us stress that, by the decomposition $\mathbb{R}^{n}=\Omega_{\text {in }} \cup \Gamma \cup \Omega_{\mathrm{ex}}, \Omega_{\text {in }} \equiv \Omega$, $\Omega_{\text {ex }}:=\mathbb{R}^{n} \backslash \bar{\Omega}$, one has $\Delta^{\circ}=\Delta_{\text {in }}^{\min } \oplus \Delta_{\mathrm{ex}}^{\min }$ and so one could obtain all selfadjoint extensions of $\Delta^{\circ}$ corresponding to separating boundary conditions by using the results (obtained by Grubb in 24, building on previous work by Birman, Krĕ̌n and Višik, see [13, 28, 39]) providing the whole family of self-adjoint extensions of $\Delta_{\text {in/ex }}^{\min }$; however such construction, broadened to include all self-adjoint extensions, would lead to a Kreĭn-type formula giving the resolvent difference between an extension $\widehat{\Delta}$ and the direct sum of the interior and exterior Dirichlet Laplacians $\Delta_{\mathrm{in}}^{D} \oplus \Delta_{\mathrm{ex}}^{D}$. This is not the right operator since we are interested in the study of the scattering system $(\widehat{\Delta}, \Delta)$, where $\Delta$ denotes the free Laplacian on the whole $\mathbb{R}^{n} ;$ whenever one considers semi-transparent boundary conditions (as the ones considered in [9] and in Section 6, Examples 6.4 and 6.5), or boundary conditions assigned only on $\Sigma \subset \Gamma$ (see Section 7 ), the choice of the Laplacian $\Delta$ as the operator representing the free propagation is the most natural one.

The first aim of this paper is to show that the Limiting Absorbtion Principle (LAP for short) holds for an ample class of self-adjoint extensions of the symmetric operator $\Delta^{\circ}$. This is accomplished by applying abstract results due to Walter Renger (see [35] and [36]) to our Kreĭn-type resolvent formula (see Theorem 4.2). As usual, LAP implies the absence of singular continuous spectrum (see Corollary 4.7). Even if interesting by itself, the result about the validity of LAP does not show that the resolvent Krĕn formula itself survives in the limit. Such a limit KreĬn's resolvent formula is here provided in next Theorem 4.5. With such results at hands, in Section 5 we construct, for a given self-adjoint extension $\widehat{\Delta}$ of $\Delta^{\circ}$, the couple of families of generalized eigenfunctions $u_{\xi}^{ \pm}$related to the plane waves $u_{\xi}^{\circ}(x)=e^{i \xi \cdot x}$ with incoming $(+)$ or outgoing $(-)$ radiation conditions. Such eigenfunctions then allow to define the corresponding Fourier type transforms $F_{ \pm}$which diagonalize the self-adjoint extension; the wave operators for the scattering system $(\widehat{\Delta}, \Delta)$ are then given by $W_{ \pm}=F_{ \pm}^{*} F$, where $F$ denotes the ordinary Fourier transform (see Theorem 5.4). Both the eigenfunctions $u_{\xi}^{ \pm}$and the Fourier transforms $F_{ \pm}$are expressed in terms of the operator-valued Weyl functions appearing in the limit Kreĭn resolvent formula given in Theorem 4.5. Finally, in Theorem 5.6, using again the operator-valued Weyl functions, we provide the kernel (proportional to the scattering amplitude) of $1-S_{k}$, where $S_{k}$ is the on-shell scattering operator.

In Sections 6 and 7, we show that our LAP-based results can be applied to a wide class of self-adjoint operators which includes self-adjoint realizations 
of the Laplacian with Dirichlet, Neumann, Robin, $\delta$ and $\delta^{\prime}$-type boundary conditions assigned either on the whole $\Gamma$ or on a relatively open subset with Lipschitz boundary $\Sigma \subset \Gamma$. We provide a representation of the scattering matrix $S_{k}$ in terms of operator-valued Weyl functions evaluated on the traces at $(\Sigma \subset) \Gamma$ of the plane waves $u_{\xi}^{\circ}$.

Our time-independent approach has been inspired by the work by Albeverio, Brasche and Koshmanenko [3], where LAP and Lippman-Schwinger equations are studied for finite-rank singular perturbations, and can be interpreted as an extension to the case of general boundary conditions and hypersurfaces of the paper [26] by Ikebe and Shimada concerning $\delta$-type boundary conditions on a sphere (see also [38]). An alternative abstract approach, which do not use LAP but directly exploits the existence of limiting operator-valued Weyl-functions, has been developed in [10], [11] and [12] by Behrndt, Malamud and Neidhardt (the first two works concern the finite-rank case; see also [1, [4, Chapter 4]). In particular, in the recent paper [12, a representation of the scattering matrix in term of operator-valued Weyl functions is provided for couples of self-adjoint extension of a given symmetric operator under the hypothesis that their resolvent difference is trace-class. In our less abstract setting, which applies to Laplacian with boundary conditions on $(\Sigma \subset) \Gamma$, we do not need the trace-class condition and the results hold in any dimension.

Let us remark that, once LAP and a Kreĭn's limit formula have been attained (see Theorems 4.2 and 4.5), a representation formula for the scattering matrix $S_{k}$ can be obtained by using the Birman-Yafaev general scheme in stationary scattering theory (see e.g. [15], [40, [41]) together with the Birman-Kato invariance principle applied to the resolvent operators (see Remark 5.7 for more details). However we preferred to present here a less abstract proof following the classical scheme used in potential scattering theory (see e.g. 22, 5], 25, 37] and references therein).

We conclude the introduction with some remarks about our smoothness hypotheses on $\Gamma$. Such an hypothesis gives the existence of the wave operators (see [29, Theorems 4.11 and 4.12]) through asymptotic estimates on the eigenvalues of the Laplace-Beltrani operator on $\Gamma$ (see [8, Lemma 4.7]). These estimates, obtained using pseudodifferential operator techniques, require smoothness; we presume that asymptotic estimates of this kind hold under a weaker $C^{1,1}$ (or at least $C^{2}$ ) hypothesis, but we did not find any proof of that in the literature. Since our result concerning existence of LAP does not require any smoothness hypothesis, conditional on the existence of wave operators, the general results here presented hold in the case $\Gamma$ is an hypersurface of class $C^{1,1}$, as for the results presented in [29], while, as regards the explicit examples given in Section 7 considering boundary conditions on $\Sigma \subset \Gamma$, one needs more regularity (of the kind $C^{k, 1}$, where 
$k>1$ depends on the kind of boundary conditions used, see [29, Section 6]). In the series of papers [19]-[21, limited to the case in which $n=2$ and Dirichlet or Neumann boundary conditions are assigned on the whole $\Gamma$, the authors provided a resolvent formula and a representation for the scattering matrix of the same kind of the ones here given in Examples 6.1 and 6.2, only assuming that the boundary $\Gamma$ is a piecewise smooth curve. This suggests that our results, which hold for a quite larger class of boundary conditions, could be extended to include the case in which $\Gamma$ is a planar curvilinear polygon (see e.g. [17] and [23] for elliptic boundary value problem in not smooth domains).

Acknowledgments. We thank the anonymous referees for the stimulating remarks, for the useful bibliographic suggestions and in particular for inspiring Remark 5.7.

The authors were partially supported by the Austrian Science Fund (FWF):[P28971-N32]

\section{Preliminaries}

2.1. Trace maps and boundary-layer operators. Here we recall some definitions and results about Sobolev spaces on subset of $\mathbb{R}^{n}$ and single and double layer operators on their boundaries (see e.g. [32] and [30]).

Given $\Omega \subset \mathbb{R}^{n}$ open and bounded, with smooth boundary $\Gamma$, we adopt the notation: $\Omega_{\mathrm{in}}=\Omega, \Omega_{\mathrm{ex}}=\mathbb{R}^{n} \backslash \bar{\Omega}$, while $\nu$ is the exterior unit normal to $\Gamma . H^{s}\left(\mathbb{R}^{n}\right), H^{s}\left(\Omega_{\text {in }}\right), H^{s}\left(\Omega_{\mathrm{ex}}\right), H^{s}(\Gamma), s \in \mathbb{R}$, denote the usual scales of Sobolev-Hilbert spaces of function on $\mathbb{R}^{n}, \Omega_{\mathrm{in}}, \Omega_{\mathrm{ex}}$ and $\Gamma$ respectively. The zero and first-order traces on $\Gamma$ are defined on smooth functions as

$$
\gamma_{0} u=\left.u\right|_{\Gamma}, \quad \gamma_{1} u=\nu \cdot \nabla u \mid \Gamma,
$$

and extend to the bounded linear operators

$$
\gamma_{0} \in \mathrm{B}\left(H^{2}\left(\mathbb{R}^{n}\right), H^{\frac{3}{2}}(\Gamma)\right), \quad \gamma_{1} \in \mathrm{B}\left(H^{2}\left(\mathbb{R}^{n}\right), H^{\frac{1}{2}}(\Gamma)\right) .
$$

We use the symbol $\Delta$ to denote the distributional Laplacian; its restriction to $H^{2}\left(\mathbb{R}^{n}\right)$

$$
\Delta: H^{2}\left(\mathbb{R}^{n}\right) \subset L^{2}\left(\mathbb{R}^{n}\right) \rightarrow L^{2}\left(\mathbb{R}^{n}\right)
$$

gives rise to a self-adjoint operator which describes the free propagation of waves in the whole space $\mathbb{R}^{n}$; this will be our reference operator.

For $z \in \mathbb{C} \backslash(-\infty, 0]$, the single and double layer operators are defined by

$$
S L_{z}=\left(\gamma_{0}(-\Delta+\bar{z})^{-1}\right)^{*}, \quad D L_{z}=\left(\gamma_{1}(-\Delta+\bar{z})^{-1}\right)^{*},
$$

and by duality there follows

$$
S L_{z} \in \mathrm{B}\left(H^{-\frac{3}{2}}(\Gamma), L^{2}\left(\mathbb{R}^{n}\right)\right), \quad D L_{z} \in \mathrm{B}\left(H^{-\frac{1}{2}}(\Gamma), L^{2}\left(\mathbb{R}^{n}\right)\right) .
$$


The integral kernel $R_{z}(x, y)$ of the resolvent $(-\Delta+z)^{-1}, z \in \mathbb{C} \backslash(-\infty, 0]$, is given by $R_{z}(x, y)=\mathscr{G}_{z}(x-y)$, where

$$
\mathscr{G}_{z}(x)=\frac{1}{2 \pi}\left(\frac{\sqrt{z}}{2 \pi\|x\|}\right)^{\frac{n}{2}-1} K_{\frac{n}{2}-1}(\sqrt{z}\|x\|), \quad \operatorname{Re}(\sqrt{z})>0,
$$

and $K_{\alpha}$ denotes the modified Bessel functions of second kind of order $\alpha$. Thus, for $x \notin \Gamma$ and $\phi, \varphi \in L^{2}(\Gamma)$, one has

$$
S L_{z} \phi(x)=\int_{\Gamma} \mathscr{G}_{z}(x-y) \phi(y) d \sigma(y),
$$

and

$$
D L_{z} \varphi(x)=\int_{\Gamma} \nu(y) \cdot \nabla \mathscr{G}_{z}(x-y) \varphi(y) d \sigma(y),
$$

where $\sigma$ denotes the surface measure.

Let us define $\gamma \in \mathrm{B}\left(H^{2}\left(\mathbb{R}^{n}\right), H^{\frac{3}{2}}(\Gamma) \oplus H^{\frac{1}{2}}(\Gamma)\right.$ by

$$
\gamma u:=\gamma_{0} u \oplus \gamma_{1} u
$$

and, for any $z \in \mathbb{C} \backslash(-\infty, 0], G_{z} \in \mathrm{B}\left(H^{-\frac{3}{2}}(\Gamma) \oplus H^{-\frac{1}{2}}(\Gamma), L^{2}\left(\mathbb{R}^{n}\right)\right)$ by

$$
G_{z}:=\left(\gamma(-\Delta+\bar{z})^{-1}\right)^{*}
$$

equivalently

$$
G_{z}(\phi \oplus \varphi):=S L_{z} \phi+D L_{z} \varphi .
$$

For any $z \in \mathbb{C} \backslash(-\infty, 0]$ and for any $\phi \oplus \varphi \in H^{-\frac{3}{2}}(\Gamma) \oplus H^{-\frac{1}{2}}(\Gamma)$ one has $G_{z}(\phi \oplus \varphi) \in \mathcal{C}^{\infty}\left(\mathbb{R}^{n} \backslash \Gamma\right) \quad$ and $\quad\left((\Delta-z) G_{z}(\phi \oplus \varphi)\right)(x)=0, \quad x \in \mathbb{R}^{n} \backslash \Gamma$.

The one-sided trace maps

$$
\gamma_{i}^{\natural} \in \mathrm{B}\left(H^{2}\left(\Omega_{\natural}\right), H^{\frac{3}{2}-i}(\Gamma)\right), \quad \natural=\text { in, ex }, \quad i=0,1,
$$

defined on smooth (up to the boundary) functions by

$$
\gamma_{0}^{\natural} u_{\natural}=u_{\natural}\left|\Gamma, \quad \gamma_{1}^{\natural} u_{\natural}=\nu \cdot \nabla u_{\natural}\right| \Gamma, \quad \natural=\text { in, ex },
$$

can be extended to

$$
\left.\hat{\gamma}_{i}^{\natural} \in \mathrm{B}\left(H_{\Delta}^{0}\left(\Omega_{\natural}\right)\right), H^{-\frac{1}{2}-i}(\Gamma)\right), \quad \natural=\text { in, ex }, \quad i=0,1,
$$

where

$$
\begin{gathered}
H_{\Delta}^{0}\left(\Omega_{\natural}\right):=\left\{u_{\natural} \in L^{2}\left(\Omega_{\natural}\right): \Delta u_{\natural} \in L^{2}\left(\Omega_{\natural}\right)\right\}, \\
\left\|u_{\natural}\right\|_{H_{\Delta}^{0}\left(\Omega_{\natural}\right)}^{2}:=\left\|\Delta u_{\natural}\right\|_{L^{2}\left(\Omega_{\natural}\right)}^{2}+\left\|u_{\natural}\right\|_{L^{2}\left(\Omega_{\natural}\right)}^{2} .
\end{gathered}
$$

Setting $\Delta_{\natural}^{\max }:=\Delta \mid H_{\Delta}^{0}\left(\Omega_{\sharp}\right)$, by the "half" Green formula (see [30, Theorem 4.4]), one has, for any $u \in H^{1}\left(\Omega_{\natural}\right) \cap H_{\Delta}^{0}\left(\Omega_{\natural}\right)$,

$$
\left\langle-\Delta_{\natural}^{\max } u_{\natural}, u_{\natural}\right\rangle_{L^{2}\left(\Omega_{\natural}\right)}=\left\|\nabla u_{\natural}\right\|_{L^{2}\left(\Omega_{\natural}\right)}^{2}+\epsilon_{\natural}\left\langle\hat{\gamma}_{1}^{\natural} u_{\natural}, \gamma_{0}^{\natural} u_{\natural}\right\rangle_{-\frac{1}{2}, \frac{1}{2}},
$$




$$
\epsilon_{\natural}= \begin{cases}-1, & \natural=\text { in } \\ +1, & \natural=\text { ex. }\end{cases}
$$

Setting

$$
H_{\Delta}^{0}\left(\mathbb{R}^{n} \backslash \Gamma\right):=H_{\Delta}^{0}\left(\Omega_{\mathrm{in}}\right) \oplus H_{\Delta}^{0}\left(\Omega_{\mathrm{ex}}\right),
$$

the extended maps $\hat{\gamma}_{i}^{\natural}$ allow to define the bounded maps

$$
\begin{gathered}
\hat{\gamma}_{i} \in \mathrm{B}\left(H_{\Delta}^{0}\left(\mathbb{R}^{n} \backslash \Gamma\right), H^{-\frac{1}{2}-i}(\Gamma)\right), \quad i=0,1, \\
{\left[\hat{\gamma}_{i}\right] \in \mathrm{B}\left(H_{\Delta}^{0}\left(\mathbb{R}^{n} \backslash \Gamma\right), H^{-\frac{1}{2}-i}(\Gamma)\right), \quad i=0,1,}
\end{gathered}
$$

by

$$
\hat{\gamma}_{i} u:=\frac{1}{2}\left(\hat{\gamma}_{i}^{\text {in }}\left(u \mid \Omega_{\mathrm{in}}\right)+\hat{\gamma}_{i}^{\mathrm{ex}}\left(u \mid \Omega_{\mathrm{ex}}\right)\right)
$$

and

$$
\left[\hat{\gamma}_{i}\right] u=\hat{\gamma}_{i}^{\mathrm{ex}}\left(u \mid \Omega_{\mathrm{ex}}\right)-\hat{\gamma}_{i}^{\text {in }}\left(u \mid \Omega_{\text {in }}\right), \quad .
$$

Notice that the maps $\gamma_{i} \in \mathrm{B}\left(H^{2}\left(\mathbb{R}^{n} \backslash \Gamma\right), H^{\frac{3}{2}-i}(\Gamma)\right)$ defined by

$$
\gamma_{i}:=\hat{\gamma}_{i} \mid H^{2}\left(\mathbb{R}^{n} \backslash \Gamma\right), \quad i=0,1, \quad H^{2}\left(\mathbb{R}^{n} \backslash \Gamma\right):=H^{2}\left(\Omega_{\mathrm{in}}\right) \oplus H^{2}\left(\Omega_{\mathrm{ex}}\right),
$$

coincide with the ones in (2.1) when restricted to $H^{2}\left(\mathbb{R}^{n}\right)$.

The corresponding extension of the trace map $\gamma$ is

$$
\hat{\gamma} \in \mathrm{B}\left(H_{\Delta}^{0}\left(\mathbb{R}^{n} \backslash \Gamma\right), H^{-\frac{1}{2}}(\Gamma) \oplus H^{-\frac{3}{2}}(\Gamma)\right), \quad \hat{\gamma} u:=\left(\hat{\gamma}_{0} u\right) \oplus\left(\hat{\gamma}_{1} u\right),
$$

while the related jump map is

$$
[\hat{\gamma}] \in \mathrm{B}\left(H_{\Delta}^{0}\left(\mathbb{R}^{n} \backslash \Gamma\right), H^{-\frac{3}{2}}(\Gamma) \oplus H^{-\frac{1}{2}}(\Gamma)\right), \quad[\hat{\gamma}] u=\left(-\left[\hat{\gamma}_{1}\right] u\right) \oplus\left(\left[\hat{\gamma}_{0}\right] u\right) .
$$

Using the definition (2.6) and the mapping properties of the layer operators (see [29, Section 3.4] and the references therein), it results

$$
G_{z} \in \mathrm{B}\left(H^{-\frac{3}{2}}(\Gamma) \oplus H^{-\frac{1}{2}}(\Gamma), H_{\Delta}^{0}\left(\mathbb{R}^{n} \backslash \Gamma\right)\right)
$$

and so $[\hat{\gamma}] G_{z} \in \mathrm{B}\left(H^{-\frac{3}{2}}(\Gamma) \oplus H^{-\frac{1}{2}}(\Gamma)\right)$. More precisely, by the well known jumps relations for the layer operators, one has

$$
[\hat{\gamma}] G_{z}=1_{H^{-\frac{3}{2}}(\Gamma) \oplus H^{-\frac{1}{2}}(\Gamma)} .
$$


LIMITING ABSORPTION PRINCIPLE, EIGENFUNCTIONS AND SCATTERING

2.2. Weighted spaces. We now introduce the family of weighted spaces $L_{\sigma}^{2}\left(\mathbb{R}^{n}\right)$ and $H_{\sigma}^{2}\left(\mathbb{R}^{n}\right)$, defined, for any $\sigma \in \mathbb{R}$, by

$$
\begin{gathered}
L_{\sigma}^{2}\left(\mathbb{R}^{n}\right):=\left\{u \in L_{\mathrm{loc}}^{2}\left(\mathbb{R}^{n}\right):\|u\|_{L_{\sigma}^{2}\left(\mathbb{R}^{n}\right)}<+\infty\right\}, \\
H_{\sigma}^{2}\left(\mathbb{R}^{n}\right):=\left\{u \in H_{\mathrm{loc}}^{2}\left(\mathbb{R}^{n}\right):\|u\|_{H_{\sigma}^{2}\left(\mathbb{R}^{n}\right)}<+\infty\right\}, \\
\|u\|_{L_{\sigma}^{2}\left(\mathbb{R}^{n}\right)}^{2}:=\int_{\mathbb{R}^{n}}\left(1+\|x\|^{2}\right)^{\sigma}|u(x)|^{2} d x \\
\|u\|_{H_{\sigma}^{2}\left(\mathbb{R}^{n}\right)}^{2}:=\|u\|_{L_{\sigma}^{2}\left(\mathbb{R}^{n}\right)}^{2}+\sum_{1 \leq i \leq n}\left\|\partial_{x_{i}} u\right\|_{L_{\sigma}^{2}\left(\mathbb{R}^{n}\right)}^{2}+\sum_{1 \leq i, j \leq n}\left\|\partial_{x_{i} x_{j}}^{2} u\right\|_{L_{\sigma}^{2}\left(\mathbb{R}^{n}\right)}^{2} .
\end{gathered}
$$

The spaces $L_{\sigma}^{2}\left(\Omega_{\text {in }}\right), L_{\sigma}^{2}\left(\Omega_{\mathrm{ex}}\right)$ and $H_{\sigma}^{2}\left(\Omega_{\text {in }}\right), H_{\sigma}^{2}\left(\Omega_{\mathrm{ex}}\right)$ are defined in a similar way. Since $\Omega$ is bounded, one has $L_{\sigma}^{2}\left(\Omega_{\text {in }}\right)=L^{2}\left(\Omega_{\text {in }}\right), H_{\sigma}^{2}\left(\Omega_{\text {in }}\right)=H^{2}\left(\Omega_{\text {in }}\right)$, the equalities holding in the Banach space sense; thus

$$
L_{\sigma}^{2}\left(\mathbb{R}^{n}\right)=L^{2}\left(\Omega_{\text {in }}\right) \oplus L_{\sigma}^{2}\left(\Omega_{\mathrm{ex}}\right)
$$

and

$$
H_{\sigma}^{2}\left(\mathbb{R}^{n} \backslash \Gamma\right):=H_{\sigma}^{2}\left(\Omega_{\mathrm{in}}\right) \oplus H_{\sigma}^{2}\left(\Omega_{\mathrm{ex}}\right)=H^{2}\left(\Omega_{\mathrm{in}}\right) \oplus H_{\sigma}^{2}\left(\Omega_{\mathrm{ex}}\right) .
$$

Then the trace operators can be extended to $H_{\sigma}^{2}\left(\mathbb{R}^{n} \backslash \Gamma\right), \sigma<0$, by

$$
\gamma_{0}^{\mathrm{ex}} u_{\mathrm{ex}}:=\gamma_{0}^{\mathrm{ex}}\left(\chi u_{\mathrm{ex}}\right), \quad \gamma_{1}^{\mathrm{ex}} u_{\mathrm{ex}}:=\gamma_{1}^{\mathrm{ex}}\left(\chi u_{\mathrm{ex}}\right),
$$

where $\chi$ belongs to $\mathcal{C}_{\text {comp }}^{\infty}\left(\Omega^{c}\right)$ and $\chi=1$ on a neighborhood of $\Gamma$.

Remark 2.1. In the following, we use the shorthand notation $\langle\cdot, \cdot\rangle$ to denote both the dualities $\left(H^{-s_{1}}(\Gamma) \oplus H^{-s_{2}}(\Gamma)\right)-\left(H^{s_{1}}(\Gamma) \oplus H^{s_{2}}(\Gamma)\right)$ and $L_{-\sigma}^{2}\left(\mathbb{R}^{n}\right)-L_{\sigma}^{2}\left(\mathbb{R}^{n}\right)$.

\section{SElF-ADJoint LAplace operators With BOUNDARY CONDITIONS ON HYPERSURFACES}

Let us consider the restriction $\Delta \mid \operatorname{ker}(\gamma)$. Since the kernel of $\gamma$ is dense in $L^{2}\left(\mathbb{R}^{n}\right), \Delta \mid \operatorname{ker}(\gamma)$ is densely defined, closed and symmetric. Following the construction developed in 29] (to which we refer for more details and proofs), we next provide all the self-adjoint extensions of $\Delta \mid \operatorname{ker}(\gamma)$. The adjoint operator $(\Delta \mid \operatorname{ker}(\gamma))^{*}$ identifies with

$$
\operatorname{dom}\left((\Delta \mid \operatorname{ker}(\gamma))^{*}\right)=H_{\Delta}^{0}\left(\mathbb{R}^{n} \backslash \Gamma\right), \quad(\Delta \mid \operatorname{ker}(\gamma))^{*}=\Delta_{\mathrm{in}}^{\max } \oplus \Delta_{\mathrm{ex}}^{\max } .
$$

An alternative representation of $(\Delta \mid \operatorname{ker}(\gamma))^{*}$ is given by (see [29, Lemma 2.3 and Lemma 4.2])

$$
\begin{aligned}
& \operatorname{dom}\left((\Delta \mid \operatorname{ker}(\gamma))^{*}\right) \\
= & \left\{u=u_{\circ}+G(\phi \oplus \varphi): u_{\circ} \in H^{2}\left(\mathbb{R}^{n}\right), \phi \oplus \varphi \in H^{-\frac{3}{2}}(\Gamma) \oplus H^{-\frac{1}{2}}(\Gamma)\right\} \\
\equiv & \left\{u \in L^{2}\left(\mathbb{R}^{n}\right): u_{\circ}:=\left(u+S L\left[\hat{\gamma}_{1}\right] u-D L\left[\hat{\gamma}_{0}\right] u\right) \in H^{2}\left(\mathbb{R}^{n}\right)\right\},
\end{aligned}
$$

$$
(\Delta \mid \operatorname{ker}(\gamma))^{*} u=\Delta u_{\circ}+G(\phi \oplus \varphi)=\Delta u-\left[\hat{\gamma}_{1}\right] u \delta_{\Gamma}-\left[\hat{\gamma}_{0}\right] u \nu \cdot \nabla \delta_{\Gamma},
$$


where $G:=G_{1}$ and the Schwartz distribution $\delta_{\Gamma}$ is defined by $\delta_{\Gamma}(u):=$ $\int_{\Gamma} u(x) d \sigma(x)$.

Given an orthogonal projection $\Pi: H^{\frac{3}{2}}(\Gamma) \oplus H^{\frac{1}{2}}(\Gamma) \rightarrow H^{\frac{3}{2}}(\Gamma) \oplus H^{\frac{1}{2}}(\Gamma)$, the dual map $\Pi^{\prime}: H^{-\frac{3}{2}}(\Gamma) \oplus H^{-\frac{1}{2}}(\Gamma) \rightarrow H^{-\frac{3}{2}}(\Gamma) \oplus H^{-\frac{1}{2}}(\Gamma)$ is an orthogonal projection as well and $\operatorname{ran}\left(\Pi^{\prime}\right)=\operatorname{ran}(\Pi)^{\prime}$. We say that the densely defined linear operator

$$
\Theta: \operatorname{dom}(\Theta) \subseteq \operatorname{ran}(\Pi)^{\prime} \rightarrow \operatorname{ran}(\Pi)
$$

is self-adjoint whenever $\Theta=\Theta^{\prime}$. Let the unitary maps $\Lambda^{s}$ represent the duality mappings on $H^{s / 2}(\Gamma)$ onto $H^{-s / 2}(\Gamma)$; an explicit representation of $\Lambda^{s}$ is given by $\Lambda^{s}=\left(-\Delta_{L B}+1\right)^{s / 2}$, where $\Delta_{L B}$ denotes the LaplaceBeltrami operator on $\Gamma$. Equivalently $\Theta$ is self-adjoint whenever the operator $\tilde{\Theta}=\Theta\left(\Lambda^{3} \oplus \Lambda\right), \operatorname{dom}(\tilde{\Theta})=\left(\Lambda^{3} \oplus \Lambda\right)^{-1} \operatorname{dom}(\Theta)$, is a self-adjoint operator in the Hilbert space $\operatorname{ran}(\Pi)$.

We define the operator-valued Weyl function

$$
\mathbb{C} \backslash(-\infty, 0] \ni z \mapsto M_{z} \in \mathrm{B}\left(H^{-\frac{3}{2}}(\Gamma) \oplus H^{-\frac{1}{2}}(\Gamma) ; H^{\frac{3}{2}}(\Gamma) \oplus H^{\frac{1}{2}}(\Gamma)\right)
$$

by $M_{z}:=\gamma\left(G-G_{z}\right)$, i.e., using the block operator notation,

$$
M_{z}=\left[\begin{array}{ll}
\gamma_{0}\left(S L-S L_{z}\right) & \gamma_{0}\left(D L-D L_{z}\right) \\
\gamma_{1}\left(S L-S L_{z}\right) & \gamma_{1}\left(D L-D L_{z}\right)
\end{array}\right]
$$

Given the couple $(\Pi, \Theta)$, $\Pi$ an orthogonal projector and $\Theta$ self-adjoint, define the set

$$
Z_{\Pi, \Theta}:=\left\{z \in \mathbb{C} \backslash(-\infty, 0]: \Theta+\Pi M_{z} \Pi^{\prime} \in \mathrm{B}\left(\operatorname{ran}(\Pi), \operatorname{ran}(\Pi)^{\prime}\right)\right\} .
$$

All self-adjoint extensions of $\Delta \mid \operatorname{ker}(\gamma)$ are provided by the following theorem (see [29, Theorem 4.4]):

Theorem 3.1. Any self-adjoint extension of $\Delta \mid \operatorname{ker}(\gamma)$ is of the kind

$$
\Delta_{\Pi, \Theta}=(\Delta \mid \operatorname{ker}(\gamma))^{*} \mid \operatorname{dom}\left(\Delta_{\Pi, \Theta}\right),
$$

where $\Pi: H^{\frac{3}{2}}(\Gamma) \oplus H^{\frac{1}{2}}(\Gamma) \rightarrow H^{\frac{3}{2}}(\Gamma) \oplus H^{\frac{1}{2}}(\Gamma)$ is an orthogonal projection, $\Theta: \operatorname{dom}(\Theta) \subseteq \operatorname{ran}(\Pi)^{\prime} \rightarrow \operatorname{ran}(\Pi)$ is a self-adjoint operator and

$$
\operatorname{dom}\left(\Delta_{\Pi, \Theta}\right)
$$$$
:=\left\{u=u_{\circ}+G(\phi \oplus \varphi): u_{\circ} \in H^{2}\left(\mathbb{R}^{n}\right), \phi \oplus \varphi \in \operatorname{dom}(\Theta), \Pi \gamma u_{\circ}=\Theta(\phi \oplus \varphi)\right\}
$$$$
=\left\{u \in H_{\Delta}^{0}\left(\mathbb{R}^{n} \backslash \Gamma\right):[\hat{\gamma}] u \in \operatorname{dom}(\Theta), \Pi \gamma\left(u+S L\left[\hat{\gamma}_{1}\right] u-D L\left[\hat{\gamma}_{0}\right] u\right)=\Theta[\hat{\gamma}] u\right\}
$$

Moreover $Z_{\Pi, \Theta}$ is not void, $\mathbb{C} \backslash \mathbb{R} \subseteq Z_{\Pi, \Theta} \subseteq \rho\left(\Delta_{\Pi, \Theta}\right)$, and the resolvent of the self-adjoint extension $\Delta_{\Pi, \Theta}$ is given by the Krĕrn's type formula

$$
\begin{aligned}
& \left(-\Delta_{\Pi, \Theta}+z\right)^{-1} \\
= & (-\Delta+z)^{-1}+G_{z} \Pi^{\prime}\left(\Theta+\Pi M_{z} \Pi^{\prime}\right)^{-1} \Pi \gamma(-\Delta+z)^{-1}, \quad z \in Z_{\Pi, \Theta} .
\end{aligned}
$$


Remark 3.2. Let us notice that the $\Pi^{\prime}$ 's appearing in formula (3.4) act there merely as the inclusion map $\Pi^{\prime}: \operatorname{ran}(\Pi)^{\prime} \rightarrow H^{-3 / 2}(\Gamma) \oplus H^{-1 / 2}(\Gamma)$. This means that one does not need to know $\Pi^{\prime}$ explicitly: it suffices to know the subspace $\operatorname{ran}\left(\Pi^{\prime}\right)=\operatorname{ran}(\Pi)^{\prime}$.

Given the self-adjoint operator $\Theta: \operatorname{dom}(\Theta) \subseteq \operatorname{ran}(\Pi)^{\prime} \rightarrow \operatorname{ran}(\Pi)$, we now introduce the following assumptions:

$$
\begin{gathered}
\operatorname{dom}(\Theta) \subseteq H^{s_{1}}(\Gamma) \oplus H^{s_{2}}(\Gamma), \quad s_{1}>-\frac{3}{2}, \quad s_{2}>-\frac{1}{2} \\
\operatorname{dom}(\Theta) \subseteq H^{\frac{1}{2}}(\Gamma) \oplus H^{\frac{3}{2}}(\Gamma)
\end{gathered}
$$

and

$$
\operatorname{dom}\left(f_{\tilde{\Theta}}\right) \subseteq H^{\frac{5}{2}}(\Gamma) \oplus H^{\frac{3}{2}}(\Gamma),
$$

where $f_{\tilde{\Theta}}$ is sesquilinear form associated to the self-adjoint operator in $\operatorname{ran}(\Pi)$ defined by $\tilde{\Theta}:=\Theta\left(\Lambda^{3} \oplus \Lambda\right)$.

The next result gives informations on the spectrum and scattering of $\Delta_{\Pi, \Theta}$; for the proof of such results we refer to [29, Lemma 4.10, Corollary 4.12 and Remark 4.14].

Theorem 3.3. Suppose Theorem 3.1 holds. Then:

1) assumption (3.5) implies

$$
\sigma_{\text {ess }}\left(\Delta_{\Pi, \Theta}\right)=(-\infty, 0] ;
$$

2) either assumption (3.6) or (3.7) gives the existence and completeness of the wave operators

$$
W_{ \pm}:=s^{-} \lim _{t \rightarrow \pm \infty} e^{-i t \Delta_{\Pi, \Theta}} e^{i t \Delta}, \quad \tilde{W}_{ \pm}:=s^{-} \lim _{t \rightarrow \pm \infty} e^{-i t \Delta} e^{i t \Delta_{\Pi, \Theta}} P_{a c}
$$

i.e. the limits exists everywhere w.r.t. strong convergence, $\operatorname{ran}\left(W_{ \pm}\right)=$ $L^{2}\left(\mathbb{R}^{n}\right)_{a c}, \operatorname{ran}\left(\tilde{W}_{ \pm}\right)=L^{2}\left(\mathbb{R}^{n}\right)$ and $W_{ \pm}^{*}=\tilde{W}_{ \pm}$, where $L^{2}\left(\mathbb{R}^{n}\right)_{a c}$ denotes the absolutely continuous subspace of $L^{2}\left(\mathbb{R}^{n}\right)$ with respect to $\Delta_{\Pi, \Theta}$ and $P_{a c}$ is the corresponding orthogonal projector. This then implies

$$
\sigma_{a c}\left(\Delta_{\Pi, \Theta}\right)=(-\infty, 0] .
$$

Remark 3.4. Let us remark that hypothesis (3.6) holds in the case of global boundary conditions, i.e. assigned on whole boundary $\Gamma$ (see Section 6), whereas hypothesis (3.7) holds in the case of local ones, i.e. assigned on $\Sigma \subset \Gamma$ (see Section 7).

Remark 3.5. Let us notice that the apparent discrepancy between the indices in (3.6) and (3.7) is due to the fact that the first one applies to operators acting between the dual pair $\left(\operatorname{ran}(\Pi)^{\prime}, \operatorname{ran}(\Pi)\right)$ whereas the second one concerns sesquilinear forms in the space $\operatorname{ran}(\Pi)$; when written in terms of $\tilde{\Theta}$, condition (3.6) reads as $\operatorname{dom}(\tilde{\Theta}) \subseteq H^{\frac{7}{2}}(\Gamma) \oplus H^{\frac{5}{2}}(\Gamma)$. 
Under hypothesis (3.6), it is possible to introduce an alternative description of $\Delta_{\Pi, \Theta}($ see $[29$, Corollary 4.8]):

Corollary 3.6. Let $\Delta_{\Pi, \Theta}$ be defined according to Theorem 3.1] with $\Theta$ fulfilling (3.6). Define

$$
B_{\Theta}:=\Theta+\Pi \gamma G \Pi^{\prime}: \operatorname{dom}(\Theta) \subseteq \operatorname{ran}(\Pi)^{\prime} \rightarrow \operatorname{ran}(\Pi) .
$$

Then

$$
\operatorname{dom}\left(\Delta_{\Pi, \Theta}\right)=\left\{u \in H^{2}\left(\mathbb{R}^{n} \backslash \Gamma\right):[\gamma] u \in \operatorname{dom}(\Theta), \Pi \gamma u=B_{\Theta}[\gamma] u\right\},
$$

and, whenever $z \in Z_{\Pi, \Theta}$,

$$
\begin{aligned}
& \left(-\Delta_{\Pi, \Theta}+z\right)^{-1} \\
= & (-\Delta+z)^{-1}+G_{z} \Pi^{\prime}\left(B_{\Theta}-\Pi \gamma G_{z} \Pi^{\prime}\right)^{-1} \Pi \gamma(-\Delta+z)^{-1} .
\end{aligned}
$$

The results contained in Theorem 3.3 do not exclude the presence of negative eigenvalues embedded in the essential spectrum, an information that is relevant for the issues to be treated in the next sections. However, since the singular perturbations defining $\Delta_{\Pi, \Theta}$ are compactly supported, an easy application of the unique continuation principle and Rellich's estimate give criteria for the absence of such eigenvalues. For successive notational convenience let us pose

$$
E_{\Pi, \Theta}^{-}:=\left\{\lambda \in(-\infty, 0): \lambda \notin \sigma_{p}\left(\Delta_{\Pi, \Theta}\right)\right\},
$$

so that absence of negative eigenvalues is equivalent to $E_{\Pi, \Theta}^{-}=(-\infty, 0)$.

Theorem 3.7. Let $\Gamma_{0} \subseteq \Gamma$ be a closed set such that $\operatorname{supp}(\phi) \cup \operatorname{supp}(\varphi) \subseteq \Gamma_{0}$ for any $\phi \oplus \varphi \in \operatorname{dom}(\Theta) \subseteq \operatorname{ran}(\Pi)^{\prime}$. If the open set $\mathbb{R}^{n} \backslash \Gamma_{0}$ is connected, then $E_{\Pi, \Theta}^{-}=(-\infty, 0)$.

Proof. Suppose that there exist $\lambda \in(-\infty, 0)$ and $u_{\lambda} \in \operatorname{dom}\left(\Delta_{\Pi, \Theta}\right) \subseteq$ $L^{2}\left(\mathbb{R}^{n}\right) \cap H_{l o c}^{2}\left(\mathbb{R}^{n} \backslash \Gamma_{0}\right)$ such that $\Delta_{\Pi, \Theta} u_{\lambda}=\lambda u_{\lambda}$. Then, by (3.1), $\Delta u_{\lambda}(x)=$ $\lambda u_{\lambda}(x)$ for a.e. $x \in \mathbb{R}^{n} \backslash \Gamma_{0}$. Thus, by the unique continuation principle (see e.g. [34, Theorem XIII.63]), $u_{\lambda}=0$ a.e. whenever $u_{\lambda}$ vanishes in the neighborhood of a single point $x_{\circ} \in \mathbb{R}^{n} \backslash \Gamma_{0}$. By (3.1) again, $(\Delta-\lambda) u_{\lambda}=0$ outside some sufficiently large ball $B$ containing $\Omega_{\text {in }}$. Thus, by Rellich's estimate, one gets $u_{\lambda} \mid B^{c}=0$ (see e.g. [31, Corollary 4.8]) and the proof is done.

Remark 3.8. Obviously, in the case $\Gamma_{0}=\Gamma$, one has that $\mathbb{R}^{n} \backslash \Gamma=\Omega_{\text {in }} \cup \Omega_{\text {ex }}$ is not connected. However, if $\Omega_{\mathrm{ex}}$ is connected then, by the same kind of reasonings as in the proof of Theorem 3.7, one gets $u_{\lambda} \mid \Omega_{\mathrm{ex}}=0$. Thus, if the boundary conditions appearing in $\operatorname{dom}\left(\Delta_{\Pi, \Theta}\right)$ are such that

$$
u\left|\Omega_{\mathrm{ex}}=0,(\Delta u-\lambda u)\right| \Omega_{\mathrm{in}}=0, u \in \operatorname{dom}\left(\Delta_{\Pi, \Theta}\right) \quad \Longrightarrow \quad u \mid \Omega_{\text {in }}=0,
$$


then $E_{\Pi, \Theta}^{-}=(-\infty, 0)$. For example, two cases where this hypothesis holds are the $\delta$ - and $\delta^{\prime}$-interactions on $\Gamma$ which corresponds to the semitransparent boundary conditions

$$
\left[\gamma_{0}\right] u=0, \quad \alpha \gamma_{0} u=\left[\gamma_{1}\right] u
$$

and

$$
\left[\gamma_{1}\right] u=0, \quad \beta \gamma_{1} u=\left[\gamma_{0}\right] u
$$

respectively (see Subsections 6.4 and 6.5).

\section{The Limiting Absorption Principle}

We begin by recalling the limiting absorption principle for the self-adjoint operator representing the free Laplacian $\Delta: H^{2}\left(\mathbb{R}^{n}\right) \subseteq L^{2}\left(\mathbb{R}^{n}\right) \rightarrow L^{2}\left(\mathbb{R}^{n}\right)$ (see e.g. [2, Section 4]):

Theorem 4.1. For any $k \in \mathbb{R} \backslash\{0\}$ and for any $\alpha>\frac{1}{2}$, the limits

$$
R_{-k^{2}}^{ \pm}:=\lim _{\epsilon \downarrow 0}\left(-\Delta-\left(k^{2} \pm i \epsilon\right)\right)^{-1}
$$

exist in $\mathrm{B}\left(L_{\alpha}^{2}\left(\mathbb{R}^{n}\right), H_{-\alpha}^{2}\left(\mathbb{R}^{n}\right)\right)$. Setting $\mathbb{C}_{ \pm}:=\{z \in \mathbb{C}: \pm \operatorname{Im}(z)>0\}$ and

$$
R_{z}^{ \pm}:= \begin{cases}(-\Delta+z)^{-1}, & z \in \mathbb{C}_{ \pm} \\ R_{\lambda}^{ \pm}, & \lambda \in(-\infty, 0),\end{cases}
$$

the maps $z \mapsto R_{z}^{ \pm}$are continuous on $\mathbb{C}_{ \pm} \cup(-\infty, 0)$ to $\mathrm{B}\left(L_{\alpha}^{2}\left(\mathbb{R}^{n}\right), H_{-\alpha}^{2}\left(\mathbb{R}^{n}\right)\right)$.

The existence of the resolvent's limits on the continuous spectrum have been discussed in [35, [36] for a wide class of operators including singular perturbations. The general results there provided allow to prove, in our case, a limiting absorption principle for $\Delta_{\Pi, \Theta}$ :

Theorem 4.2. Let $\Delta_{\Pi, \Theta}$ be defined as in Theorem 3.1 and assume that it is bounded from above and that (3.5) holds true. Then $(-\infty, 0) \cap \sigma_{p}\left(\Delta_{\Pi, \Theta}\right)$ is a (possibly empty) discrete set of eigenvalues of finite multiplicity and the limits

$$
R_{\Pi, \Theta,-k^{2}}^{ \pm}:=\lim _{\epsilon \downarrow 0}\left(-\Delta_{(\Pi, \Theta)}-\left(k^{2} \pm i \epsilon\right)\right)^{-1}
$$

exist in $\mathrm{B}\left(L_{\alpha}^{2}\left(\mathbb{R}^{n}\right), L_{-\alpha}^{2}\left(\mathbb{R}^{n}\right)\right)$ for all $\alpha>\frac{1}{2}$ and for all $k \in \mathbb{R} \backslash\{0\}$ such that $-k^{2} \in E_{\Pi, \Theta}^{-} \cdot$

Proof. Let us at first show that the following four assumptions hold true for any $z \in \mathbb{C} \backslash(-\infty, 0]$ :

$$
\begin{gathered}
(-\Delta+z)^{-1} \in \mathrm{B}\left(L_{\alpha}^{2}\left(\mathbb{R}^{n}\right)\right), \\
\left(-\Delta_{\Pi, \Theta}+z\right)^{-1} \in \mathrm{B}\left(L_{\alpha}^{2}\left(\mathbb{R}^{n}\right)\right),
\end{gathered}
$$




$$
(-\Delta+z)^{-1}-\left(-\Delta_{\Pi, \Theta}+z\right)^{-1} \in \mathfrak{S}_{\infty}\left(L^{2}\left(\mathbb{R}^{n}\right), L_{\beta}^{2}\left(\mathbb{R}^{n}\right)\right), \quad \beta>2 \alpha,
$$

and for all compact subset $K \subset(0,+\infty)$ there exists a constant $c_{K}>0$ such that, for any $k^{2} \in K$,

(4.6) $\forall u \in L_{2 \alpha}^{2}\left(\mathbb{R}^{n}\right) \cap \operatorname{ker}\left(R_{-k^{2}}^{+}-R_{-k^{2}}^{-}\right), \quad\left\|R_{-k^{2}}^{ \pm} u\right\|_{L^{2}\left(\mathbb{R}^{n}\right)} \leq c_{K}\|u\|_{L_{2 \alpha}^{2}\left(\mathbb{R}^{n}\right)}$.

By [34, Lemma 1, page 170], for all $\sigma \in \mathbb{R}$ one has

$$
(-\Delta+z)^{-1} \in \mathrm{B}\left(L_{\sigma}^{2}\left(\mathbb{R}^{n}\right)\right) .
$$

Therefore (4.3) holds true (see also [36, Lemma 5.2 and Remark 5.1]).

Introducing the equivalent norm in $H_{\sigma}^{2}\left(\mathbb{R}^{n}\right)$

$$
|u|_{H_{\sigma}^{2}\left(\mathbb{R}^{n}\right)}:=\int_{\mathbb{R}^{n}}\left(1+\|x\|^{2}\right)^{\sigma}|(-\Delta+1) u(x)|^{2} d x,
$$

by

$$
\begin{aligned}
\left|(-\Delta+z)^{-1} u\right|_{H_{\sigma}^{2}\left(\mathbb{R}^{n}\right)}^{2} & =\left\|(-\Delta+1)(-\Delta+z)^{-1} u\right\|_{L_{\sigma}^{2}\left(\mathbb{R}^{n}\right)}^{2} \\
& \leq\|u\|_{L_{\sigma}^{2}\left(\mathbb{R}^{n}\right)}^{2}+|1-z|\left\|(-\Delta+z)^{-1} u\right\|_{L_{\sigma}^{2}\left(\mathbb{R}^{n}\right)}^{2}
\end{aligned}
$$

and by (4.7), one gets

$$
(-\Delta+z)^{-1} \in \mathrm{B}\left(L_{\sigma}^{2}\left(\mathbb{R}^{n}\right), H_{\sigma}^{2}\left(\mathbb{R}^{n}\right)\right) .
$$

Let $\chi \in \mathcal{C}_{\text {comp }}^{\infty}\left(\mathbb{R}^{n}\right)$ such that $\chi \mid \tilde{\Omega}=1, \tilde{\Omega} \supset \bar{\Omega}$. Then the map $u \mapsto \chi u$ belongs to $\mathrm{B}\left(H_{\sigma}^{2}\left(\mathbb{R}^{n}\right), H^{2}\left(\mathbb{R}^{n}\right)\right)$ and so, since $\gamma(\chi u)=\gamma u$, by (4.8) one gets

$$
\gamma(-\Delta+z)^{-1} \in \mathrm{B}\left(L_{\sigma}^{2}\left(\mathbb{R}^{n}\right), H^{\frac{3}{2}}(\Gamma) \oplus H^{\frac{1}{2}}(\Gamma)\right) .
$$

and, by duality,

$$
G_{z}=\left(\gamma(-A+\bar{z})^{-1}\right)^{\prime} \in \mathrm{B}\left(H^{-\frac{3}{2}}(\Gamma) \oplus H^{-\frac{1}{2}}(\Gamma), L_{-\sigma}^{2}\left(\mathbb{R}^{n}\right)\right) .
$$

Then, using (4.9) and (4.10) with $\sigma=\alpha$ and with $\sigma=-\alpha$ respectively, (4.4) follows from (3.4) and (4.7).

Assumption (3.5) implies that $\operatorname{ran}\left(\left(\Theta+\Pi M_{z} \Pi^{\prime}\right)^{-1}\right) \subseteq H^{s_{1}}(\Gamma) \oplus H^{s_{2}}(\Gamma)$. Thus, by the compact embedding $H^{s_{1}}(\Gamma) \oplus H^{s_{2}}(\Gamma) \hookrightarrow H^{-3 / 2}(\Gamma) \oplus H^{-1 / 2}(\Gamma)$, one gets

$$
\left(\Theta+\Pi M_{z} \Pi^{\prime}\right)^{-1} \in \mathfrak{S}_{\infty}\left(\operatorname{ran}(\Pi), \operatorname{ran}\left(\Pi^{\prime}\right)\right)
$$

(see the proof of Lemma 4.10 in [29] for more details). Therefore, by (3.4), using (4.9) and (4.10) with $\sigma=0$ and $\sigma=-\beta$ respectively, one obtains (4.5).

Finally, (4.6) holds true by [7, Corollary 5.7(b)].

Assumptions (4.3)-(4.6) permit us to apply the abstract results provided in [35]: hypothesis (T1) and (E1) in [35, page 175] corresponds to our (4.1), (4.6) and (4.5) respectively; then, by [35, Proposition 4.2], the latters imply hypotheses (LAP) and (E) in [35, page 166], i.e. (4.1) again and

$$
(-\Delta+z)^{-1}-\left(-\Delta_{\Pi, \Theta}+z\right)^{-1} \in \mathfrak{S}_{\infty}\left(L_{-\alpha}^{2}\left(\mathbb{R}^{n}\right), L_{\alpha}^{2}\left(\mathbb{R}^{n}\right)\right),
$$


and hypothesis $(\mathrm{T})$ in [35, page 168], a technical variant of (4.6). By [35, Theorem 3.5], these last hypotheses, together with the assumption that $-\Delta_{\Pi, \Theta}$ is bounded from below and (4.3)-(4.4) (i.e. hypothesis (OP) in [35, page 165]), finally give the content of the theorem.

Remark 4.3. Since the map defined in (4.9) has closed range (it is surjective), $G_{z} \in \mathrm{B}\left(H^{-\frac{3}{2}}(\Gamma) \oplus H^{-\frac{1}{2}}(\Gamma), L_{-\sigma}^{2}\left(\mathbb{R}^{n}\right)\right)$ defined in (4.10) is injective and has closed range by the closed range theorem. Hence, by [27, Theorem 5.2 , page 231], for any $z \in \rho(A)$ there exists $c_{z}>0$ such that

$$
\left\|G_{z}(\phi \oplus \varphi)\right\|_{L_{-\sigma}^{2}\left(\mathbb{R}^{n}\right)}^{2} \geq c_{z}\left(\|\phi\|_{H^{-\frac{3}{2}(\Gamma)}}^{2}+\|\varphi\|_{H^{-\frac{1}{2}(\Gamma)}}^{2}\right)
$$

for all $\phi \oplus \varphi \in H^{-\frac{3}{2}}(\Gamma) \oplus H^{-\frac{1}{2}}(\Gamma)$.

Lemma 4.4. For any $k \in \mathbb{R} \backslash\{0\}$ and for any $\alpha>\frac{1}{2}$, the limits

$$
G_{-k^{2}}^{ \pm}:=\lim _{\epsilon \downarrow 0} G_{-\left(k^{2} \pm i \epsilon\right)}
$$

exist in $\mathrm{B}\left(H^{-\frac{3}{2}}(\Gamma) \oplus H^{-\frac{1}{2}}(\Gamma), L_{-\alpha}^{2}\left(\mathbb{R}^{n}\right)\right)$ and

$$
\begin{gathered}
G_{-k^{2}}^{ \pm}=G_{z}+\left(z+k^{2}\right) R_{-k^{2}}^{ \pm} G_{z}, \quad z \in \mathbb{C} \backslash(-\infty, 0], \\
\left(G_{-k^{2}}^{ \pm}\right)^{\prime}=\gamma R_{-k^{2}}^{\mp} .
\end{gathered}
$$

The function $G_{-k^{2}}^{ \pm}(\phi \oplus \varphi)$ solves, in the distribution space $\mathscr{D}^{\prime}\left(\mathbb{R}^{n} \backslash \Gamma\right)$ and for any $\phi \oplus \varphi \in H^{-\frac{3}{2}}(\Gamma) \oplus H^{-\frac{1}{2}}(\Gamma)$, the equation

$$
\left(\Delta+k^{2}\right) G_{-k^{2}}^{ \pm}(\phi \oplus \varphi)=0 .
$$

Moreover there exist $c_{k^{2}}^{ \pm}>0$ such that

$$
\left\|G_{-k^{2}}^{ \pm}(\phi \oplus \varphi)\right\|_{L_{-\alpha}^{2}\left(\mathbb{R}^{n}\right)}^{2} \geq c_{k^{2}}^{ \pm}\left(\|\phi\|_{H^{-\frac{3}{2}(\Gamma)}}^{2}+\|\varphi\|_{H^{-\frac{1}{2}(\Gamma)}}^{2}\right) \text {. }
$$

Proof. Let $\chi \in C_{\mathrm{comp}}^{\infty}\left(\mathbb{R}^{n}\right)$ such that $\chi \mid \tilde{\Omega}=1, \tilde{\Omega} \supset \bar{\Omega}$. Then the map $u \mapsto \chi u$ belongs to $\mathrm{B}\left(H_{-\alpha}^{2}\left(\mathbb{R}^{n}\right), H^{2}\left(\mathbb{R}^{n}\right)\right)$ and so, by Theorem 4.1, the limits

$$
\gamma R_{-k^{2}}^{ \pm}=\lim _{\epsilon \downarrow 0} \gamma\left(-\Delta-\left(k^{2} \pm i \epsilon\right)\right)^{-1}
$$

exist in $\mathrm{B}\left(L_{\alpha}^{2}\left(\mathbb{R}^{n}\right), H^{\frac{3}{2}}(\Gamma) \oplus H^{\frac{1}{2}}(\Gamma)\right)$. Then the relations

$$
\begin{aligned}
& G_{-\left(k^{2} \pm i \epsilon\right)}=\left(\gamma\left(-\Delta-k^{2} \mp i \epsilon\right)\right)^{\prime} \\
= & G_{z}+\left(z+k^{2} \mp i \epsilon\right)\left(-\Delta-\left(k^{2} \pm i \epsilon\right)\right)^{-1} G_{z}
\end{aligned}
$$

(see [33, Lemma 2.1]), and $G_{z} \in \mathrm{B}\left(H^{-\frac{1}{2}}(\Gamma) \oplus H^{-\frac{3}{2}}(\Gamma), L_{\alpha}^{2}(\mathbb{R})\right)$ (use (4.10) with $\sigma=-\alpha$ ), give (4.13), (4.14) and (4.15). 
Since

$$
\lim _{\epsilon \downarrow 0}\left\|\left(\Delta+\left(k^{2} \pm i \epsilon\right)\right) u-\left(\Delta+k^{2}\right) u\right\|_{L_{\alpha}^{2}\left(\mathbb{R}^{n}\right)}=0, \quad u \in H_{\alpha}^{2}\left(\mathbb{R}^{n}\right),
$$

one has

$$
R_{-k^{2}}^{ \pm}\left(-\Delta-k^{2}\right) u=u, \quad u \in H_{\alpha}^{2}\left(\mathbb{R}^{n}\right) .
$$

Thus, for any test function $u \in \mathscr{D}\left(\mathbb{R}^{n} \backslash \Gamma\right) \equiv C_{\text {comp }}^{\infty}\left(\mathbb{R}^{n} \backslash \Gamma\right) \subset H_{\alpha}^{2}\left(\mathbb{R}^{n}\right)$, one obtains

$$
\begin{aligned}
& \left\langle\left(\Delta+k^{2}\right) G_{-k^{2}}^{ \pm}(\phi \oplus \varphi), u\right\rangle=\left\langle G_{-k^{2}}^{ \pm}(\phi \oplus \varphi),\left(\Delta+k^{2}\right) u\right\rangle \\
= & \left\langle\left(\gamma R_{-k^{2}}^{\mp}\right)^{\prime}(\phi \oplus \varphi),\left(\Delta+k^{2}\right) u\right\rangle=-\left\langle\phi \oplus \varphi, \gamma R_{-k^{2}}^{ \pm}\left(\Delta+k^{2}\right) u\right\rangle \\
= & -\langle\phi \oplus \varphi, \gamma u\rangle=0 .
\end{aligned}
$$

By (4.18) and the surjectivity of $\gamma: H^{2}\left(\mathbb{R}^{n}\right) \rightarrow H^{\frac{3}{2}}(\Gamma) \oplus H^{\frac{1}{2}}(\Gamma)$, the map

$$
\gamma R_{-k^{2}}^{ \pm}: L_{\alpha}^{2}\left(\mathbb{R}^{n}\right) \rightarrow H^{\frac{3}{2}}(\Gamma) \oplus H^{\frac{1}{2}}(\Gamma)
$$

is surjective: given $\phi \oplus \varphi \in H^{\frac{3}{2}}(\Gamma) \oplus H^{\frac{1}{2}}(\Gamma)$ one has $\gamma R_{-k^{2}}^{ \pm} v=\phi \oplus \varphi$, where $v=-\left(\Delta+k^{2}\right) \chi u$ and $\gamma \chi u=\gamma u=\phi \oplus \varphi, u \in H^{2}\left(\mathbb{R}^{n}\right)$. Therefore, by the closed range theorem, the range of $G_{-k^{2}}^{ \pm}$is closed; since $G_{-k^{2}}^{ \pm}$is injective (it is the dual of a surjective map), [27, Theorem 5.2, page 231] gives (4.16).

While interesting, Theorem 4.2 gives no answer to the obvious question: "does KreĬn's formula survive in the limit $\epsilon \downarrow 0$ ?" That is given by the following

Theorem 4.5. Under the assumptions of Theorem 4.2, for any $-k^{2} \in$ $E_{\Pi, \Theta}^{-}$, the limits

$$
\begin{gathered}
M_{-k^{2}}^{ \pm}:=\lim _{\epsilon \downarrow 0} M_{-\left(k^{2} \pm i \epsilon\right)}, \\
L_{\Pi, \Theta,-k^{2}}^{ \pm}:=\lim _{\epsilon \downarrow 0}\left(\Theta+\Pi M_{-\left(k^{2} \pm i \epsilon\right)} \Pi^{\prime}\right)^{-1}
\end{gathered}
$$

exist in $\mathrm{B}\left(H^{-\frac{3}{2}}(\Gamma) \oplus H^{-\frac{1}{2}}(\Gamma), H^{\frac{3}{2}}(\Gamma) \oplus H^{\frac{1}{2}}(\Gamma)\right)$ and $\mathrm{B}\left(\operatorname{ran}(\Pi), \operatorname{ran}\left(\Pi^{\prime}\right)\right)$ respectively and

$$
M_{-k^{2}}^{ \pm}=M_{z}-\left(z+k^{2}\right) \gamma R_{-k^{2}}^{ \pm} G_{z}, \quad z \in \mathbb{C} \backslash(-\infty, 0] .
$$

The linear operator $\Theta+\Pi M_{-k^{2}}^{ \pm} \Pi^{\prime}$ has a bounded inverse

$$
L_{\Pi, \Theta,-k^{2}}^{ \pm}=\left(\Theta+\Pi M_{-k^{2}}^{ \pm} \Pi^{\prime}\right)^{-1}
$$

and

$$
R_{\Pi, \Theta,-k^{2}}^{ \pm}-R_{-k^{2}}^{ \pm}=G_{-k^{2}}^{ \pm} \Pi^{\prime}\left(\Theta+\Pi M_{-k^{2}}^{ \pm} \Pi^{\prime}\right)^{-1} \Pi \gamma R_{-k^{2}}^{ \pm}
$$


Moreover the map

$$
z \mapsto R_{\Pi, \Theta, z}^{ \pm}:= \begin{cases}\left(-\Delta_{\Pi, \Theta}+z\right)^{-1}, & z \in \mathbb{C}_{ \pm} \\ R_{\Pi, \Theta, \lambda}^{ \pm}, & \lambda \in E_{\Pi, \Theta}^{-}\end{cases}
$$

is continuous on $\mathbb{C}_{ \pm} \cup E_{\Pi, \Theta}^{-}$to $\mathrm{B}\left(L_{\alpha}^{2}\left(\mathbb{R}^{n}\right), L_{-\alpha}^{2}\left(\mathbb{R}^{n}\right)\right)$.

Proof. By [33, equation (5)], the operator family $M_{z}, z \in \mathbb{C} \backslash(-\infty, 0]$, satisfies the identity

$$
M_{-\left(k^{2} \pm i \epsilon\right)}=M_{z}-\left(z+k^{2} \mp i \epsilon\right) \gamma\left(-\Delta-\left(k^{2} \pm i \epsilon\right)\right)^{-1} G_{z} .
$$

Since, by (4.10), $G_{z} \in \mathrm{B}\left(H^{-\frac{1}{2}}(\Gamma) \oplus H^{-\frac{3}{2}}(\Gamma), L_{\alpha}^{2}\left(\mathbb{R}^{n}\right)\right)$, the norm convergence of $M_{-\left(k^{2} \pm i \epsilon\right)}$ to $M_{-k^{2}}^{ \pm}$is consequence of Theorem 4.1. This gives (4.19) and (4.21).

By Theorem 4.2, the limits

$$
\lim _{\epsilon \downarrow 0} G_{-\left(k^{2} \pm i \epsilon\right)} \Pi^{\prime}\left(\Theta+\Pi M_{-\left(k^{2} \pm i \epsilon\right)} \Pi^{\prime}\right)^{-1} \Pi \gamma\left(-\Delta-\left(k^{2} \pm i \epsilon\right)\right)^{-1}
$$

exist in $\mathrm{B}\left(L_{\alpha}^{2}\left(\mathbb{R}^{n}\right), L_{-\alpha}^{2}\left(\mathbb{R}^{n}\right)\right)$ and, by Lemma 4.4, the limits

$$
G_{-k^{2}}^{ \pm}=\lim _{\epsilon \downarrow 0} G_{-\left(k^{2} \pm i \epsilon\right)}
$$

and

$$
\left(G_{-k^{2}}^{\mp}\right)^{\prime}=\gamma R_{-k^{2}}^{ \pm}=\lim _{\epsilon \downarrow 0} \gamma\left(-\Delta-\left(k^{2} \pm i \epsilon\right)\right)^{-1}
$$

exist in $\mathrm{B}\left(H^{-\frac{1}{2}}(\Gamma) \oplus H^{-\frac{3}{2}}(\Gamma), L_{-\alpha}^{2}\left(\mathbb{R}^{n}\right)\right)$ and $\mathrm{B}\left(L_{\alpha}^{2}\left(\mathbb{R}^{n}\right), H^{\frac{1}{2}}(\Gamma) \oplus H^{\frac{3}{2}}(\Gamma)\right)$ respectively. According to (4.12) and (4.16), there exist $\tilde{c}_{k^{2}}^{ \pm}>0$ such that, for all $\epsilon>0$,

$$
\begin{gathered}
\left\|G_{-\left(k^{2} \pm i \epsilon\right)} \Pi^{\prime}\left(\Theta+\Pi M_{-\left(k^{2} \pm i \epsilon\right)} \Pi^{\prime}\right)^{-1} \Pi \gamma\left(-\Delta-\left(k^{2} \pm i \epsilon\right)\right)^{-1} u\right\|_{L_{-\alpha}^{2}\left(\mathbb{R}^{n}\right)}^{2} \\
\geq \tilde{c}_{k^{2}}^{ \pm}\left(\left\|\left(\Theta+\Pi M_{-\left(k^{2} \pm i \epsilon\right)} \Pi^{\prime}\right)^{-1} \Pi \gamma\left(-\Delta-\left(k^{2} \pm i \epsilon\right)\right)^{-1} u\right\|_{H^{-\frac{3}{2}}(\Gamma) \oplus H^{-\frac{1}{2}}(\Gamma)}^{2}\right) .
\end{gathered}
$$

Let $\|\cdot\|$ denote the operator norm in $\mathrm{B}(X, Y)$, the Hilbert spaces $X$ and $Y$ varying according to the case. Then, by (4.24), one has

$$
\sup _{\epsilon>0}\left\|\left(\Theta+\Pi M_{-\left(k^{2} \pm i \epsilon\right)} \Pi^{\prime}\right)^{-1} \Pi \gamma\left(-\Delta-\left(k^{2} \pm i \epsilon\right)\right)^{-1}\right\|<+\infty .
$$

and, by duality

$$
\sup _{\epsilon>0}\left\|G_{-\left(k^{2} \pm i \epsilon\right)} \Pi^{\prime}\left(\Theta+\Pi M_{-\left(k^{2} \pm i \epsilon\right)} \Pi^{\prime}\right)^{-1}\right\|<+\infty .
$$


Thus, by

$$
\begin{aligned}
& \left\|G_{-\left(k^{2} \pm i \epsilon\right)} \Pi^{\prime}\left(\Theta+\Pi M_{-\left(k^{2} \pm i \epsilon\right)} \Pi^{\prime}\right)^{-1} \Pi\left(\gamma\left(-\Delta-\left(k^{2} \pm i \epsilon\right)\right)^{-1}-\gamma R_{-k^{2}}^{ \pm}\right)\right\| \\
\leq & \left(\sup _{\epsilon>0}\left\|G_{-\left(k^{2} \pm i \epsilon\right)} \Pi^{\prime}\left(\Theta+\Pi M_{-\left(k^{2} \pm i \epsilon\right)} \Pi^{\prime}\right)^{-1}\right\|\right) \\
& \times\left\|\gamma\left(-\Delta-\left(k^{2} \pm i \epsilon\right)\right)^{-1}-\gamma R_{-k^{2}}^{ \pm}\right\|
\end{aligned}
$$

and by (4.24), (4.26), one has that the limits

$$
\lim _{\epsilon \downarrow 0} G_{-\left(k^{2} \pm i \epsilon\right)} \Pi^{\prime}\left(\Theta+\Pi M_{-\left(k^{2} \pm i \epsilon\right)} \Pi^{\prime}\right)^{-1} \Pi \gamma R_{-k^{2}}^{ \pm}
$$

exist in $\mathrm{B}\left(L_{\alpha}^{2}\left(\mathbb{R}^{n}\right), L_{-\alpha}^{2}\left(\mathbb{R}^{n}\right)\right)$ and coincide with the ones given in (4.24). Since the map $\gamma R_{-k^{2}}^{ \pm}$is surjective (see the end of the proof of Lemma 4.4), by [27, Theorem 5.2, page 231] there exists $\hat{c}_{-k^{2}}^{ \pm}>0$ such that

$$
\forall u \in \operatorname{ker}\left(\gamma R_{-k^{2}}^{ \pm}\right)^{\perp}, \quad\left\|\gamma R_{-k^{2}}^{ \pm} u\right\|_{H^{\frac{1}{2}}(\Gamma) \oplus H^{\frac{3}{2}(\Gamma)}} \geq \hat{c}_{-k^{2}}^{ \pm}\|u\|_{L_{\alpha}^{2}\left(\mathbb{R}^{n}\right)} .
$$

Setting $\Phi_{\epsilon}:=G_{-\left(k^{2} \pm i \epsilon\right)} \Pi^{\prime}\left(\Theta+\Pi M_{-\left(k^{2} \pm i \epsilon\right)} \Pi^{\prime}\right)^{-1} \Pi$, we have

$$
\begin{array}{r}
\sup _{\{0\} \neq \phi \oplus \varphi \in H^{\frac{1}{2}}(\Gamma) \oplus H^{\frac{3}{2}}(\Gamma)} \frac{\left\|\left(\Phi_{\epsilon_{1}}-\Phi_{\epsilon_{2}}\right) \phi \oplus \varphi\right\|_{L_{-\alpha}^{2}\left(\mathbb{R}^{n}\right)}}{\|\phi \oplus \varphi\|_{H^{\frac{1}{2}}(\Gamma) \oplus H^{\frac{3}{2}}(\Gamma)}} \\
=\sup _{u \in \operatorname{ker}\left(\gamma R_{-k^{2}}^{ \pm}\right)^{\perp}} \frac{\left\|\left(\Phi_{\epsilon_{1}}-\Phi_{\epsilon_{2}}\right) \gamma R_{-k^{2}}^{ \pm} u\right\|_{L_{-\alpha}^{2}\left(\mathbb{R}^{n}\right)}}{\left\|\gamma R_{-k^{2}}^{ \pm} u\right\|_{H^{\frac{1}{2}}(\Gamma) \oplus H^{\frac{3}{2}}(\Gamma)}} \\
\leq \sup _{u \in \operatorname{ker}\left(\gamma R_{-k^{2}}^{ \pm}\right)^{\perp}} \frac{\left\|\left(\Phi_{\epsilon_{1}}-\Phi_{\epsilon_{2}}\right) \gamma R_{-k^{2}}^{ \pm} u\right\|_{L_{-\alpha}^{2}\left(\mathbb{R}^{n}\right)}}{\hat{c}_{-k^{2}}^{ \pm}\|u\|_{L_{\alpha}^{2}\left(\mathbb{R}^{n}\right)}} .
\end{array}
$$

Hence, by (4.27), the limits

$$
\lim _{\epsilon \downarrow 0} G_{-\left(k^{2} \pm i \epsilon\right)} \Pi^{\prime}\left(\Theta+\Pi M_{-\left(k^{2} \pm i \epsilon\right)} \Pi^{\prime}\right)^{-1} \Pi
$$

exist in $\mathrm{B}\left(\operatorname{ran}(\Pi), L_{-\alpha}^{2}\left(\mathbb{R}^{n}\right)\right)$ and, by duality, the limits

$$
\lim _{\epsilon \downarrow 0}\left(\Theta+\Pi M_{-\left(k^{2} \pm i \epsilon\right)} \Pi^{\prime}\right)^{-1} \Pi \gamma\left(-\Delta-\left(k^{2} \pm i \epsilon\right)\right)^{-1}
$$

exist in $\mathrm{B}\left(L_{\alpha}^{2}\left(\mathbb{R}^{n}\right), \operatorname{ran}\left(\Pi^{\prime}\right)\right)$. By (4.12) and (4.16), it results

$$
\begin{aligned}
& \left\|G_{-\left(k^{2} \pm i \epsilon\right)} \Pi^{\prime}\left(\Theta+\Pi M_{-\left(k^{2} \pm i \epsilon\right)} \Pi^{\prime}\right)^{-1} \Pi(\phi \oplus \varphi)\right\|_{L_{-\alpha}^{2}\left(\mathbb{R}^{n}\right)}^{2} \\
\geq & \tilde{c}_{k^{2}}^{ \pm}\left(\left\|\left(\Theta+\Pi M_{-\left(k^{2} \pm i \epsilon\right)} \Pi^{\prime}\right)^{-1} \Pi(\phi \oplus \varphi)\right\|_{H^{-\frac{3}{2}}(\Gamma) \oplus H^{-\frac{1}{2}(\Gamma)}}^{2}\right)
\end{aligned}
$$

and so (4.28) gives

$$
\sup _{\epsilon>0}\left\|\left(\Theta+\Pi M_{-\left(k^{2} \pm i \epsilon\right)} \Pi^{\prime}\right)^{-1}\right\|<+\infty .
$$


Therefore, by (4.29), one gets that the limits

$$
\lim _{\epsilon \downarrow 0}\left(\Theta+\Pi M_{-\left(k^{2} \pm i \epsilon\right)} \Pi^{\prime}\right)^{-1} \Pi \gamma R_{-k^{2}}^{ \pm}
$$

exist in $\mathrm{B}\left(L_{\alpha}^{2}\left(\mathbb{R}^{n}\right), \operatorname{ran}\left(\Pi^{\prime}\right)\right)$ and coincide with the ones given by (4.29). Since $\gamma R_{-k^{2}}^{ \pm}$is surjective, proceeding as above one gets the existence of the limits

$$
\lim _{\epsilon \downarrow 0}\left(\Theta+\Pi M_{-\left(k^{2} \pm i \epsilon\right)} \Pi^{\prime}\right)^{-1}
$$

with respect to the operator norm in $B\left(\operatorname{ran}(\Pi), \operatorname{ran}\left(\Pi^{\prime}\right)\right)$. Finally, taking the limit $\epsilon \downarrow 0$ in the identities

$$
\begin{aligned}
& \left(\Theta+\Pi M_{-\left(k^{2} \pm i \epsilon\right)} \Pi^{\prime}\right)^{-1}\left(\Theta+\Pi M_{-\left(k^{2} \pm i \epsilon\right)} \Pi^{\prime}\right) \\
= & \left(\Theta+\Pi M_{-\left(k^{2} \pm i \epsilon\right)} \Pi^{\prime}\right)\left(\Theta+\Pi M_{-\left(k^{2} \pm i \epsilon\right)} \Pi^{\prime}\right)^{-1}=1,
\end{aligned}
$$

one gets

$\left(\Theta+\Pi M_{-k^{2}}^{ \pm} \Pi^{\prime}\right)^{-1}\left(\Theta+\Pi M_{-k^{2}}^{ \pm} \Pi^{\prime}\right)=\left(\Theta+\Pi M_{-k^{2}}^{ \pm} \Pi^{\prime}\right)\left(\Theta+\Pi M_{-k^{2}}^{ \pm} \Pi^{\prime}\right)^{-1}=1$.

By Theorem 4.1, the map $z \mapsto \gamma R_{z}^{ \pm}$is continuous on $\mathbb{C}_{ \pm} \cup(-\infty, 0)$ to $\mathrm{B}\left(L_{\alpha}^{2}\left(\mathbb{R}^{n}\right), H^{\frac{3}{2}}(\Gamma) \oplus H^{\frac{1}{2}}(\Gamma)\right)$; by duality, $z \mapsto G_{z}^{ \pm}=\left(\gamma R_{z}^{ \pm}\right)^{\prime}$ is continuos on $\mathbb{C}_{ \pm} \cup(-\infty, 0)$ to $\mathrm{B}\left(H^{-\frac{3}{2}}(\Gamma) \oplus H^{-\frac{1}{2}}(\Gamma), L_{-\alpha}^{2}\left(\mathbb{R}^{n}\right)\right)$. By (4.21),$z \mapsto M_{z}^{ \pm}$ is continuos on $\mathbb{C}_{ \pm} \cup(-\infty, 0)$ to $\mathrm{B}\left(H^{\frac{3}{2}}(\Gamma) \oplus H^{\frac{1}{2}}(\Gamma), H^{-\frac{3}{2}}(\Gamma) \oplus H^{-\frac{1}{2}}(\Gamma)\right)$ and so, by (4.30), $z \mapsto\left(\Theta+\Pi M_{z}^{ \pm} \Pi^{\prime}\right)^{-1}$ is continuos on $\mathbb{C}_{ \pm} \cup(-\infty, 0)$ to $\mathrm{B}\left(\operatorname{ran}(\Pi), \operatorname{ran}\left(\Pi^{\prime}\right)\right)$. In conclusion $z \mapsto R_{\Pi, \Theta, z}^{ \pm}$is continuous on $\mathbb{C}_{ \pm} \cup(-\infty, 0)$ to $\mathrm{B}\left(L_{\alpha}^{2}\left(\mathbb{R}^{n}\right), L_{-\alpha}^{2}\left(\mathbb{R}^{n}\right)\right)$.

Corollary 4.6. If, in addition to hypotheses in Theorem 4.5, hypothesis (3.6) holds, then the Krein type formula (4.23) can be re-written as

$$
R_{\Pi, \Theta,-k^{2}}^{ \pm}-R_{-k^{2}}^{ \pm}=G_{-k^{2}}^{ \pm} \Pi^{\prime}\left(B_{\Theta}-\Pi \gamma G_{-k^{2}}^{ \pm} \Pi^{\prime}\right)^{-1} \Pi \gamma R_{-k^{2}}^{ \pm} .
$$

Proof. By hypothesis (3.6), one has

$$
\operatorname{ran}\left(G_{z}\right) \mid \operatorname{dom}(\Theta) \subseteq H^{2}\left(\mathbb{R}^{n} \backslash \Gamma\right) .
$$

Then, by (4.14) and (4.17), the operator

$$
\gamma G_{-k^{2}}^{ \pm}: \operatorname{dom}(\Theta) \rightarrow H^{\frac{3}{2}}(\Gamma) \oplus H^{\frac{1}{2}}(\Gamma)
$$

is well-defined and, for any $\phi \oplus \varphi \in \operatorname{dom}(\Theta)$, the limits

$$
\gamma G_{-k^{2}}^{ \pm}(\phi \oplus \varphi)=\lim _{\epsilon \downarrow 0} \gamma G_{-\left(k^{2} \pm i \epsilon\right)}(\phi \oplus \varphi)
$$


exists in $H^{\frac{3}{2}}(\Gamma) \oplus H^{\frac{1}{2}}(\Gamma)$. Thus, for any $\phi \oplus \varphi \in \operatorname{dom}(\Theta)$ and for any $-k^{2} \in E_{\Pi, \Theta}^{-}$, one has

$$
\begin{aligned}
& \left(\Theta+\Pi M_{-k^{2}}^{ \pm} \Pi^{\prime}\right)(\phi \oplus \varphi)=\lim _{\epsilon \downarrow 0}\left(\Theta+\Pi M_{-\left(k^{2} \pm i \epsilon\right)} \Pi^{\prime}\right)(\phi \oplus \varphi) \\
= & \lim _{\epsilon \downarrow 0}\left(B_{\Theta}-\Pi \gamma G_{-\left(k^{2} \pm i \epsilon\right)} \Pi^{\prime}\right)(\phi \oplus \varphi)=\left(B_{\Theta}-\Pi \gamma G_{-k^{2}}^{ \pm} \Pi^{\prime}\right)(\phi \oplus \varphi) .
\end{aligned}
$$

Corollary 4.7. Under the hypotheses in Theorem 4.2, $\Delta_{\Pi, \Theta}$ has empty singular continuous spectrum, i.e.

$$
L^{2}\left(\mathbb{R}^{n}\right)=L^{2}\left(\mathbb{R}^{n}\right)_{a c} \oplus L^{2}\left(\mathbb{R}^{n}\right)_{p p},
$$

where $L^{2}\left(\mathbb{R}^{n}\right)_{\text {ac }}$ and $L^{2}\left(\mathbb{R}^{n}\right)_{p p}$ denote the absolutely continuous and pure point subspaces of $L^{2}\left(\mathbb{R}^{n}\right)$ with respect to $\Delta_{\Pi, \Theta}$.

Proof. We follow standard arguments (see e.g. [2, Theorem 6.1]): let $E_{\lambda}$ the spectral resolution of $\Delta_{\Pi, \Theta}$ and let $u \in L_{\alpha}^{2}\left(\mathbb{R}^{n}\right) \cap L^{2}\left(\mathbb{R}^{n}\right)_{p p}^{\perp}$. Then, for any compact interval $[a, b] \subset E_{\Pi, \Theta}^{-}$one has, by Stone's formula, by the continuity of $z \mapsto R_{\Pi, \Theta, z}^{ \pm}$and by Lebesgue's dominated converge theorem,

$$
\begin{aligned}
& \left\langle\left(E_{b}-E_{a}\right) u, u\right\rangle_{L^{2}\left(\mathbb{R}^{n}\right)} \\
= & \lim _{\epsilon \downarrow 0} \frac{1}{2 \pi i} \int_{a}^{b}\left\langle\left(\left(-\Delta_{\Pi, \Theta}+\lambda-i \epsilon\right)^{-1}-\left(-\Delta_{\Pi, \Theta}+\lambda+i \epsilon\right)^{-1}\right) u, u\right\rangle d \lambda \\
= & \frac{1}{2 \pi i} \int_{a}^{b}\left\langle\left(R_{\Pi, \Theta, \lambda}^{-}-R_{\Pi, \Theta, \lambda}^{+}\right) u, u\right\rangle d \lambda
\end{aligned}
$$

so that $\left\langle E_{\lambda} u, u\right\rangle_{L^{2}\left(\mathbb{R}^{n}\right)}$ is differentiable on $E_{\Pi, \Theta}^{-}$and

$$
\frac{d}{d \lambda}\left\langle E_{\lambda} u, u\right\rangle_{L^{2}\left(\mathbb{R}^{n}\right)}=\frac{1}{2 \pi i}\left\langle\left(R_{\Pi, \Theta, \lambda}^{-}-R_{\Pi, \Theta, \lambda}^{+}\right) u, u\right\rangle
$$

for all $u \in L_{\alpha}^{2}\left(\mathbb{R}^{n}\right)$. Since it is known that the set of functions for which $\left\langle E_{\lambda} u, u\right\rangle_{L^{2}\left(\mathbb{R}^{n}\right)}$ is differentiable is a closed set, in conclusion $\left\langle E_{\lambda} u, u\right\rangle_{L^{2}\left(\mathbb{R}^{n}\right)}$ is differentiable for any $u \in L^{2}\left(\mathbb{R}^{n}\right)_{p p}^{\perp}$.

\section{EIGENFUNCTION EXPANSION AND THE SCATTERING MATRIX}

All over this section we suppose that the assumptions in Theorem 4.2 hold true. We then consider the extension $\tilde{\Delta}_{\Pi, \Theta}$ of the self-adjoint operator

$$
\Delta_{\Pi, \Theta}: \operatorname{dom}\left(\Delta_{\Pi, \Theta}\right) \subseteq L^{2}\left(\mathbb{R}^{n}\right) \rightarrow L^{2}\left(\mathbb{R}^{n}\right)
$$

to the larger space $L_{-\alpha}^{2}\left(\mathbb{R}^{n}\right), \alpha>0$, given by

$$
\begin{gathered}
\tilde{\Delta}_{\Pi, \Theta}: \operatorname{dom}\left(\tilde{\Delta}_{\Pi, \Theta}\right) \subseteq L_{-\alpha}^{2}\left(\mathbb{R}^{n}\right) \rightarrow L_{-\alpha}^{2}\left(\mathbb{R}^{n}\right), \\
\tilde{\Delta}_{\Pi, \Theta} u:=\Delta u_{\circ}+G(\phi \oplus \varphi)=\Delta u-\left[\hat{\gamma}_{1}\right] u \delta_{\Gamma}-\left[\hat{\gamma}_{0}\right] u \nu \cdot \nabla \delta_{\Gamma},
\end{gathered}
$$


$\operatorname{dom}\left(\tilde{\Delta}_{\Pi, \Theta}\right)$

$:=\left\{u=u_{\circ}+G(\phi \oplus \varphi): u_{\circ} \in H_{-\alpha}^{2}\left(\mathbb{R}^{n}\right), \phi \oplus \varphi \in \operatorname{dom}(\Theta), \Pi \gamma u_{\circ}=\Theta(\phi \oplus \varphi)\right\}$.

By Theorem 4.2 and (3.1) one has

$$
\operatorname{graph}\left(\tilde{\Delta}_{\Pi, \Theta}\right) \cap\left(L^{2}\left(\mathbb{R}^{n}\right) \oplus L^{2}\left(\mathbb{R}^{n}\right)\right)=\operatorname{graph}\left(\Delta_{\Pi, \Theta}\right) .
$$

Such an operator $\tilde{\Delta}_{\Pi, \Theta}$ allows the introduction of the generalized eigenfunctions of $\Delta_{\Pi, \Theta}$ :

Theorem 5.1. Let $\tilde{u}_{k} \in L_{-\alpha}^{2}\left(\mathbb{R}^{n}\right) \backslash\{0\}$ be a generalized eigenfunction of $\Delta_{\Pi, \Theta}$ with eigenvalue $-k^{2} \in E_{\Pi, \Theta}^{-}$, i.e. $\tilde{u}_{k}$ belongs to $\operatorname{dom}\left(\tilde{\Delta}_{\Pi, \Theta}\right)$ and solves the equation

$$
\left(\tilde{\Delta}_{\Pi, \Theta}+k^{2}\right) \tilde{u}_{k}=0 .
$$

Then

$$
\tilde{u}_{k}=u_{k}+G_{-k^{2}}^{ \pm} \Pi^{\prime}\left(\Theta+\Pi M_{-k^{2}}^{ \pm} \Pi^{\prime}\right)^{-1} \Pi \gamma u_{k},
$$

where $u_{k} \in H_{-\alpha}^{2}\left(\mathbb{R}^{n}\right)$ is a generalized eigenfunction of $\Delta: H^{2}\left(\mathbb{R}^{n}\right) \subset$ $L^{2}\left(\mathbb{R}^{n}\right) \rightarrow L^{2}\left(\mathbb{R}^{n}\right)$ with eigenvalue $-k^{2}$.

Proof. Let us set $\tilde{u}_{k}=u_{\circ}+G(\phi \oplus \varphi)$, with $u_{\circ} \in H_{-\alpha}^{2}\left(\mathbb{R}^{n}\right)$ and $\phi \oplus \varphi \in$ $\operatorname{dom}(\Theta)$ such that $\Pi \gamma u_{\circ}=\Theta(\phi \oplus \varphi)$. Then $\left(\tilde{\Delta}_{\Pi, \Theta}+k^{2}\right) \tilde{u}_{k}=0$ gives

$$
\left(\Delta+k^{2}\right) u_{\circ}=-\left(1+k^{2}\right) G(\phi \oplus \varphi) .
$$

Since $\operatorname{ran}(G) \subset L_{\alpha}^{2}\left(\mathbb{R}^{n}\right)$, we can apply $R_{-k^{2}}^{ \pm}$to both sides of the above relation; thus

$$
u_{\circ}=u_{k}+\left(1+k^{2}\right) R_{-k^{2}}^{ \pm} G(\phi \oplus \varphi),
$$

where $u_{k} \in H_{-\alpha}^{2}\left(\mathbb{R}^{n}\right)$ is any solution of the equation $\left(\Delta+k^{2}\right) u_{k}=0$. Imposing the boundary conditions we obtain, by (4.21) and by $M_{1}=0$,

$$
\Pi \gamma u_{\circ}=\Pi \gamma u_{k}-\Pi M_{-k^{2}}^{ \pm}(\phi \oplus \varphi)=\Theta(\phi \oplus \varphi),
$$

i.e.

$$
\phi \oplus \varphi=\left(\Theta+\Pi M_{-k^{2}}^{ \pm} \Pi^{\prime}\right)^{-1} \Pi \gamma u_{k} .
$$

The proof is then concluded by using (4.14) with $z=1$.

Remark 5.2. Under hypothesis (3.6), by Corollary [3.6, one can alternatively define

$\operatorname{dom}\left(\tilde{\Delta}_{\Pi, \Theta}\right):=\left\{u \in L_{-\alpha}^{2}\left(\mathbb{R}^{n}\right) \cap H_{-\alpha}^{2}\left(\mathbb{R}^{n} \backslash \Gamma\right):[\gamma] u \in \operatorname{dom}(\Theta), \Pi \gamma u=B_{\Theta}[\gamma] u\right\}$

and so

$$
\tilde{u}_{k}=u_{k}+G_{-k^{2}}^{ \pm} \Pi^{\prime}\left(B_{\Theta}-\Pi \gamma G_{-k^{2}}^{ \pm} \Pi^{\prime}\right)^{-1} \Pi \gamma u_{k}
$$


Before stating the next results we recall the following definition: let $u$ solve the Helmholtz equation $\left(\Delta+k^{2}\right) u=0$ on the exterior of some bounded domain; we say that $u$ satisfies the $( \pm)$ Sommerfeld radiation condition whenever

$$
\lim _{\|x\| \rightarrow+\infty}\|x\|^{(n-1) / 2}(\hat{x} \cdot \nabla \pm i k) u(x)=0
$$

hold uniformly in $\hat{x}:=x /\|x\|$. The plus sign corresponds to an inward wave and the minus one corresponds to a outward wave.

Lemma 5.3. 1) the functions $G_{-k^{2}}^{ \pm}(\phi \oplus \varphi)$ satisfy the $( \pm)$ Sommerfeld radiation condition.

2) If $u \in \operatorname{ker}\left(\tilde{\Delta}_{\Pi, \Theta}+k^{2}\right),-k^{2} \in E_{\Pi, \Theta}^{-}$, satisfies the Sommerfeld radiation condition, then $u=0$.

Proof. By (4.15),

$$
G_{-k^{2}}^{ \pm}(\phi \oplus \varphi)=S L_{-k^{2}}^{ \pm} \phi+D L_{-k^{2}}^{ \pm} \varphi
$$

where

$$
S L_{-k^{2}}^{ \pm}:=\left(\gamma_{0} R_{-k^{2}}^{\mp}\right)^{\prime}, \quad D L_{-k^{2}}^{ \pm}:=\left(\gamma_{1} R_{-k^{2}}^{\mp}\right)^{\prime} .
$$

By (2.5) and $K_{\alpha}(z)=\frac{\pi}{2} i^{\alpha+1} H_{\alpha}^{(1)}(i z)$, where $H_{\alpha}^{(1)}$ denotes the Hankel function of first kind of order $\alpha$, it results

$\mathscr{G}_{-\left(k^{2} \pm i \epsilon\right)}(x)=\frac{i}{4}\left(\frac{\sqrt{k^{2} \mp i \epsilon}}{2 \pi\|x\|}\right)^{\frac{n}{2}-1} H_{\frac{n}{2}-1}^{(1)}\left(\sqrt{k^{2} \mp i \epsilon}\|x\|\right), \quad \operatorname{Im} \sqrt{k^{2} \mp i \epsilon}>0$.

Therefore, by $\sqrt{k^{2} \mp i \epsilon}=\mp|k|+\frac{i}{2} \frac{\epsilon}{|k|}+o(\epsilon)$ for any $k \neq 0$,

$$
\mathscr{G}_{-k^{2}}^{ \pm}(x):=\lim _{\epsilon \downarrow 0} \mathscr{G}_{-\left(k^{2} \pm i \epsilon\right)}(x)=\frac{i}{4}\left(\frac{\mp|k|}{2 \pi\|x\|}\right)^{\frac{n}{2}-1} H_{\frac{n}{2}-1}^{(1)}(\mp|k|\|x\|) .
$$

Thus, for any fixed $k \neq 0$, one gets (see e.g. [5, Appendix 1])

$$
\sup _{\epsilon>0}\left|\mathscr{G}_{-\left(k^{2} \pm i \epsilon\right)}(x)\right| \leq c\left(\|x\|^{-\frac{1}{2}(n-1)}+\|x\|^{-(n-2)}\right) .
$$

By the dominated convergence theorem, for any bounded and compactly supported $u$ and $v$,

$$
\lim _{\epsilon \downarrow 0}\left\langle u,\left(R_{-\left(k^{2} \pm i \epsilon\right)}-\tilde{R}_{-k^{2}}^{ \pm}\right) v\right\rangle_{L^{2}\left(\mathbb{R}^{n}\right)}=0,
$$

where $\tilde{R}_{-k^{2}}^{ \pm}$denotes the operator with integral kernel given by $\mathscr{G}_{-k^{2}}^{ \pm}(x-y)$. Therefore, by Theorem 4.1, $R_{-k^{2}}^{ \pm}=\tilde{R}_{-k^{2}}^{ \pm}$and so, if $\phi$ and $\varphi$ are in $L^{2}(\Gamma)$ and $x \notin \Gamma$,

$$
S L_{-k^{2}}^{ \pm} \phi(x)=\int_{\Gamma} \mathscr{G}_{-k^{2}}^{ \pm}(x-y) \phi(y) d \sigma(y)
$$


and

$$
D L_{-k^{2}}^{ \pm} \varphi(x)=\int_{\Gamma} \nu(y) \cdot \nabla_{G_{-k^{2}}^{ \pm}}^{ \pm}(x-y) \varphi(y) d \sigma(y) .
$$

Then, by the behavior of $H_{\alpha}^{(1)}(x)$ and $\frac{d}{d x} H_{\alpha}^{(1)}(x)$ as $\|x\| \rightarrow+\infty$, there follows that $S L_{-k^{2}}^{ \pm} \phi=\mathscr{G}_{-k^{2}}^{ \pm} *\left(\phi \delta_{\Gamma}\right)$ and $D L_{-k^{2}}^{ \pm} \varphi=\mathscr{G}_{-k^{2}}^{ \pm} *\left(\varphi \nu \cdot \nabla \delta_{\Gamma}\right)$ (and hence $\left.G_{-k^{2}}^{ \pm}(\phi \oplus \varphi)\right)$ satisfy the $( \pm)$ Sommerfeld radiation condition (see e.g. [18, Lemma 7, Subsection 7d, Section 8, Chapter II]).

2 ) Let us suppose that $u \neq 0$. Then, by Theorem 5.1,

$$
u=u_{k}+G_{-k^{2}}^{ \pm} \Pi^{\prime}\left(\Theta+\Pi M_{-k^{2}}^{ \pm} \Pi^{\prime}\right)^{-1} \Pi \gamma u_{k}
$$

where $u_{k} \in H_{-\alpha}^{2}\left(\mathbb{R}^{n}\right)$ is a generalized eigenfunction of $\Delta$ with eigenvalue $-k^{2}$. Then, by 1), $u$ satisfies the Sommerfeld radiation condition if and only if $u_{k}$ does. By Green's formula on the ball of radius $R$, since $\left(\Delta+k^{2}\right) u_{k}(x)=$ 0 for any $x \in \mathbb{R}^{3}$, one has

$$
\operatorname{Im}\left(\int_{\|x\|=R} \bar{u}_{k}(x) \hat{x} \cdot \nabla u_{k}(x) d \sigma(x)\right)=0 .
$$

Thus, by [30, Lemma 9.9], if $u_{k}$ satisfies the Sommerfeld radiation condition then $u_{k}(x)=0$ for any $\|x\|>R$. Since $R$ is arbitrary, this gives $u_{k}=0$, contradicting our assumption $u \neq 0$.

By Theorem 5.1 and by considering the usual family of generalized eigenfunctions $u_{\xi}^{\circ} \in H_{-\alpha}^{2}\left(\mathbb{R}^{n}\right), \alpha>\frac{n}{2}$, of $\Delta: H^{2}\left(\mathbb{R}^{n}\right) \subset L^{2}\left(\mathbb{R}^{n}\right) \rightarrow L^{2}\left(\mathbb{R}^{n}\right)$ given by the plane waves

$$
u_{\xi}^{\circ}(x):=e^{i \xi \cdot x},
$$

one obtains the two families of generalized eigenfunctions of $\Delta_{\Pi, \Theta}$ defined by

$$
u_{\xi}^{ \pm}:=u_{\xi}^{\circ}+G_{-k^{2}}^{\mp} \Pi^{\prime}\left(\Theta+\Pi M_{-k^{2}}^{\mp} \Pi^{\prime}\right)^{-1} \Pi \gamma u_{\xi}^{\circ}, \quad k=\|\xi\|, \quad-k^{2} \in E_{\Pi, \Theta}^{-} .
$$

Remark 5.4. Since $\operatorname{ran}\left(R_{-k^{2}}^{ \pm}\right) \subseteq H_{-\alpha}^{2}\left(\mathbb{R}^{n}\right)$, by (2.14) and (4.14), one has

$$
[\gamma] G_{-k^{2}}^{ \pm}=1_{H^{-\frac{3}{2}}(\Gamma) \oplus H^{-\frac{1}{2}}(\Gamma)} .
$$

Thus, since $[\gamma] u_{\xi}^{\circ}=0$, one gets

$$
[\gamma] u_{\xi}^{ \pm}=\left(\Theta+\Pi M_{-k^{2}}^{\mp} \Pi^{\prime}\right)^{-1} \Pi \gamma u_{\xi}^{\circ},
$$

and so the functions $u_{\xi}^{ \pm} \in \operatorname{dom}\left(\tilde{\Delta}_{\Pi, \Theta}\right)$ solve the Lippmann-Schwinger type equation

$$
u_{\xi}^{ \pm}=u_{\xi}^{\circ}+G_{-k^{2}}^{\mp}[\gamma] u_{\xi}^{ \pm} .
$$


Let us now define, for any $u \in L_{\alpha}^{2}\left(\mathbb{R}^{n}\right)$,

$$
\begin{aligned}
& F_{ \pm}^{\circ} u(\xi):=\frac{1}{(2 \pi)^{\frac{n}{2}}} \int_{\mathbb{R}^{n}} \overline{u_{\xi}^{ \pm}}(x) u(x) d x \\
= & \left.F u(\xi)+\frac{1}{(2 \pi)^{\frac{n}{2}}}\left\langle\left(\Theta+\Pi M_{-k^{2}}^{\mp} \Pi^{\prime}\right)^{-1} \Pi \gamma u_{\xi}^{\circ}\right), \gamma R_{-k^{2}}^{ \pm} u\right\rangle \\
= & F u(\xi)+\frac{1}{(2 \pi)^{\frac{n}{2}}}\left\langle[\gamma] u_{\xi}^{ \pm}, \gamma R_{-k^{2}}^{ \pm} u\right\rangle,
\end{aligned}
$$

where $F$ denotes the Fourier transform and $\langle\cdot, \cdot\rangle$ denotes the $\left(H^{-s_{1}}(\Gamma) \oplus\right.$ $\left.H^{-s_{2}}(\Gamma)\right)-\left(H^{s_{1}}(\Gamma) \oplus H^{s_{2}}(\Gamma)\right)$ duality. Next theorem provides the main properties of the maps $F_{ \pm}^{\circ}$ :

Theorem 5.5. 1) The $F_{ \pm}^{\circ}$ extend to bounded operators $F_{ \pm} \in \mathrm{B}\left(L^{2}\left(\mathbb{R}^{n}\right)\right)$ such that $\operatorname{ker}\left(F_{ \pm}\right)=L^{2}\left(\mathbb{R}^{n}\right)_{p p}$ and $F_{ \pm} \mid L^{2}\left(\mathbb{R}^{n}\right)_{a c}$ are unitary onto $L^{2}\left(\mathbb{R}^{n}\right)$.

2) Let $P_{a c}$ be the orthogonal projection onto $L^{2}\left(\mathbb{R}^{n}\right)_{a c}$, then

$$
\forall u \in \operatorname{dom}\left(\Delta_{\Pi, \Theta}\right), \quad\left(F_{ \pm} P_{a c} \Delta_{\Pi, \Theta} u\right)(\xi)=-\|\xi\|^{2} F_{ \pm} u(\xi) .
$$

3) Assume either (3.6) or (3.7) holds, so that the wave operators

$$
W_{ \pm}:=s-\lim _{t \rightarrow \pm \infty} e^{-i t \Delta_{\Pi, \ominus}} e^{i t \Delta}
$$

exist and are complete; then

$$
W_{ \pm}=F_{ \pm}^{*} F
$$

Proof. 1) We adapt to our framework the reasonings in [2, Section 6] (see also [25]). By (3.4), one has, for any $z \in Z_{\Pi, \Theta}$,

$$
\begin{aligned}
& \left(F\left(-\Delta_{\Pi, \Theta}+z\right)^{-1} u\right)(\xi) \\
= & \left(\|\xi\|^{2}+z\right)^{-1} F u(\xi)+\left(F G_{z} \Pi^{\prime}\left(\Theta+\Pi M_{z} \Pi^{\prime}\right)^{-1} \Pi \gamma(-\Delta+z)^{-1} u\right)(\xi) \\
= & \left(\|\xi\|^{2}+z\right)^{-1} F u(\xi) \\
& \left.+\frac{1}{(2 \pi)^{n / 2}}\left\langle\left(\Theta+\Pi M_{\bar{z}} \Pi^{\prime}\right)^{-1} \gamma(-\Delta+\bar{z})^{-1} u_{\xi}^{\circ}, \gamma(-\Delta+z)^{-1} u\right)\right\rangle \\
= & \left(\|\xi\|^{2}+z\right)^{-1} \breve{u}_{z}(\xi),
\end{aligned}
$$

where

$$
\left.\breve{u}_{z}(\xi):=F u(\xi)+\frac{1}{(2 \pi)^{n / 2}}\left\langle\left(\Theta+\Pi M_{\bar{z}} \Pi^{\prime}\right)^{-1} \gamma u_{\xi}^{\circ}, \gamma(-\Delta+z)^{-1} u\right)\right\rangle .
$$

Then, for $u \in L_{\alpha}^{2}\left(\mathbb{R}^{n}\right)$, we set

$$
\breve{u}_{-k^{2}}^{ \pm}(\xi):=\lim _{\epsilon \downarrow 0} \breve{u}_{-\left(k^{2} \pm i \epsilon\right)}(\xi) .
$$

By the Theorems 4.1 and 4.5, such a definition is well-posed and

$$
\breve{u}_{-k^{2}}^{ \pm}(\xi)=F u(\xi)+\frac{1}{(2 \pi)^{n / 2}}\left\langle\left(\Theta+\Pi M_{-k^{2}}^{\mp} \Pi^{\prime}\right)^{-1} \gamma u_{\xi}^{\circ}, \gamma R_{-k^{2}}^{ \pm} u\right\rangle,
$$


so that, for any $u \in L_{\alpha}^{2}\left(\mathbb{R}^{n}\right)$,

$$
\breve{u}_{-\|\xi\|^{2}}^{ \pm}(\xi)=F_{ \pm}^{\circ} u(\xi) .
$$

Then, one has (see [2, page 191] for the reasonings that allow the exchange $\left.\lim \int=\int \lim \right)$

$$
\begin{aligned}
& \left\langle\left(E_{b}-E_{a}\right) u, u\right\rangle_{L^{2}\left(\mathbb{R}^{n}\right)} \\
= & \lim _{\epsilon \downarrow 0} \frac{\epsilon}{\pi} \int_{a}^{b}\left\|\left(-\Delta_{\Pi, \Theta}+(\lambda \pm i \epsilon)\right)^{-1} u\right\|_{L^{2}\left(\mathbb{R}^{n}\right)}^{2} d \lambda \\
= & \left.\lim _{\epsilon \downarrow 0} \frac{\epsilon}{\pi} \int_{a}^{b} \| F\left(-\Delta_{\Pi, \Theta}+\lambda \pm i \epsilon\right)^{-1} u\right) \|_{L^{2}\left(\mathbb{R}^{n}\right)}^{2} d \lambda \\
= & \lim _{\epsilon \downarrow 0} \frac{\epsilon}{\pi} \int_{a}^{b}\left(\int_{\mathbb{R}^{n}}\left|\|\xi\|^{2}+\lambda \pm i \epsilon\right|^{-2}\left|\breve{u}_{\lambda \pm i \epsilon}(\xi)\right|^{2} d \xi\right) d \lambda \\
= & \int_{\mathbb{R}^{n}}\left(\lim _{\epsilon \downarrow 0} \frac{\epsilon}{\pi} \int_{a}^{b}\left|\|\xi\|^{2}+\lambda \pm i \epsilon\right|^{-2}\left|\breve{u}_{\lambda \pm i \epsilon}(\xi)\right|^{2} d \lambda\right) d \xi .
\end{aligned}
$$

By the known properties of the Poisson integral (see e.g. [2, equation $(6.16)])$,

$$
\begin{aligned}
\lim _{\epsilon \downarrow 0} \frac{\epsilon}{\pi} \int_{a}^{b}\left|\|\xi\|^{2}+\lambda \pm i \epsilon\right|^{-2}\left|\breve{u}_{\lambda \pm i \epsilon}(\xi)\right|^{2} d \lambda \\
\quad= \begin{cases}\left|\breve{u}_{-\|\xi\|^{2}}(\xi)\right|^{2}, & a<-\|\xi\|^{2}<b \\
0, & \text { otherwise }\end{cases}
\end{aligned}
$$

Therefore

$$
\begin{aligned}
\left\langle\left(E_{b}-E_{a}\right) u, u\right\rangle_{L^{2}\left(\mathbb{R}^{n}\right)} & =\int_{a<-\|\xi\|^{2}<b}\left|\breve{u}_{-\|\xi\|^{2}}(\xi)\right|^{2} d \xi \\
& =\int_{a<-\|\xi\|^{2}<b}\left|F_{ \pm}^{\circ} u(\xi)\right|^{2} d \xi
\end{aligned}
$$

and so, if $P_{a c}$ denotes the orthogonal projector onto $L^{2}\left(\mathbb{R}^{n}\right)_{a c}$, for any $u \in L_{\alpha}^{2}\left(\mathbb{R}^{n}\right)$ one has

$$
\left\|P_{a c} u\right\|_{L^{2}\left(\mathbb{R}^{n}\right)}^{2}=\left\langle P_{a c} u, u\right\rangle_{L^{2}\left(\mathbb{R}^{n}\right)}=\int_{\mathbb{R}^{n}}\left|F_{ \pm}^{\circ} u(\xi)\right|^{2} d \xi=\left\|F_{ \pm}^{\circ} u\right\|_{L^{2}\left(\mathbb{R}^{n}\right)}^{2} .
$$

This shows that $F_{ \pm}^{\circ}$ can be extended by continuity to a bounded map $F_{ \pm} \in \mathrm{B}\left(L^{2}\left(\mathbb{R}^{n}\right)\right.$. By (5.8), one gets $\operatorname{ker}\left(F_{ \pm}\right)=L^{2}\left(\mathbb{R}^{n}\right)_{p p}$ and $F_{ \pm}$is an isometry from $L^{2}\left(\mathbb{R}^{n}\right)_{a c}$ into $L^{2}\left(\mathbb{R}^{n}\right)$. By Theorem 3.3 , $\operatorname{ran}\left(W_{ \pm}^{*}\right)=L^{2}\left(\mathbb{R}^{n}\right)$ and so $\operatorname{ran}\left(F_{ \pm}\right)=L^{2}\left(\mathbb{R}^{n}\right)$ will be a consequence of (5.5) which will be proven below. 
2) By (5.7) and by the polarization identity, for any $u, v \in L^{2}\left(\mathbb{R}^{n}\right)_{a c}$,

$$
\left\langle E_{\lambda} u, v\right\rangle_{L^{2}\left(\mathbb{R}^{n}\right)}=\int_{-\|\xi\|^{2}<\lambda} \overline{F_{ \pm} u}(\xi) F_{ \pm} v(\xi) d \xi
$$

and so, for any $u, v \in L^{2}\left(\mathbb{R}^{n}\right)_{a c} \cap \operatorname{dom}\left(\Delta_{\Pi, \Theta}\right)$,

$$
\begin{aligned}
& \left\langle\Delta_{\Pi, \Theta} u, v\right\rangle_{L^{2}\left(\mathbb{R}^{n}\right)}=\int_{-\infty}^{0} \lambda\left\langle E_{\lambda} u, v\right\rangle_{L^{2}\left(\mathbb{R}^{n}\right)} d \lambda \\
= & -\int_{\mathbb{R}^{n}}\|\xi\|^{2} \overline{F_{ \pm} u}(\xi) F_{ \pm} v(\xi) d \xi=-\left\langle\|\cdot\|^{2} F_{ \pm} u, F_{ \pm} v\right\rangle_{L^{2}\left(\mathbb{R}^{n}\right)} .
\end{aligned}
$$

3) We equivalently show that $F_{ \pm} W_{ \pm} u=F u$ for any $u$ in the Schwartz space of rapidly decreasing functions. Let define $W_{ \pm}(t):=P_{a c} e^{-i t \Delta_{\Pi, \Theta}} e^{i t \Delta}$. Since we are assuming the existence of the strong limits which define $W_{ \pm}$, such limits can be replaced by the Abelian ones (see e.g. Corollary 14 and Lemma 15 in [6, Section 6.1.2]); therefore

$$
\begin{aligned}
& F_{ \pm} W_{ \pm} u=\lim _{\epsilon \rightarrow 0 \pm} \epsilon \int_{0}^{ \pm \infty} e^{-\epsilon t} F_{ \pm} W_{ \pm}(t) u d t \\
= & \lim _{\epsilon \rightarrow 0 \pm} \int_{0}^{ \pm \infty} e^{-\epsilon t} \frac{d}{d t} F_{ \pm} W_{ \pm}(t) u d t+F_{ \pm} u .
\end{aligned}
$$

The map $F_{ \pm}$diagonalize $P_{a c} \Delta_{\Pi, \Theta}$, thus

$$
\left(F_{ \pm} W_{ \pm}(t) u\right)(\xi)=\frac{1}{(2 \pi)^{n / 2}}\left\langle u_{\xi}^{ \pm}, e^{i t\left(\Delta+\|\xi\|^{2}\right)} u\right\rangle
$$

(here and below $\langle\cdot, \cdot\rangle$ denotes the $L_{-\alpha}^{2} L_{\alpha}^{2}$ duality). Since $\left(\Delta+\|\xi\|^{2}\right) u_{\xi}^{\circ}=0$ and $u_{\xi}^{ \pm}=u_{\xi}^{\circ}+G_{-\|\xi\|^{2}}^{\mp}[\gamma] u_{\xi}^{ \pm}$, we get

$$
\begin{aligned}
& e^{-\epsilon t} \frac{d}{d t}\left(F_{ \pm} W_{ \pm}(t) u\right)(\xi)=\frac{i e^{-\epsilon t}}{(2 \pi)^{n / 2}}\left\langle u_{\xi}^{ \pm},\left(\Delta+\|\xi\|^{2}\right) e^{i t\left(\Delta+\|\xi\|^{2}\right)} u\right\rangle \\
= & \frac{i e^{-\epsilon t}}{(2 \pi)^{n / 2}}\left(\left\langle\left(\Delta+\|\xi\|^{2}\right) u_{\xi}^{\circ}, e^{i t\left(\Delta+\|\xi\|^{2}\right)} u\right\rangle\right. \\
& \left.+\left\langle G_{-\|\xi\|^{2}}^{\mp}[\gamma] u_{\xi}^{ \pm},\left(\Delta+\|\xi\|^{2}\right) e^{i t\left(\Delta+\|\xi\|^{2}\right)} u\right\rangle\right) \\
= & \frac{i}{(2 \pi)^{n / 2}}\left\langle G_{-\|\xi\|^{2}}^{\mp}[\gamma] u_{\xi}^{ \pm},\left(\Delta+\|\xi\|^{2}\right) e^{i t\left(\Delta+\|\xi\|^{2}+i \epsilon\right)} u\right\rangle \\
= & \frac{1}{(2 \pi)^{n / 2}} \frac{d}{d t}\left\langle G_{-\|\xi\|^{2}}^{\mp}[\gamma] u_{\xi}^{ \pm},\left(\Delta+\|\xi\|^{2}\right)\left(\Delta+\|\xi\|^{2}+i \epsilon\right)^{-1} e^{i t\left(\Delta+\|\xi\|^{2}+i \epsilon\right)} u\right\rangle .
\end{aligned}
$$


Therefore, a.e. (eventually taking the limit along a subsequence)

$$
\begin{aligned}
& \left(F_{ \pm} W_{ \pm} u\right)(\xi) \\
= & \lim _{\epsilon \rightarrow 0 \pm} \int_{0}^{ \pm \infty} e^{-\epsilon t} \frac{d}{d t}\left(F_{ \pm} W_{ \pm}(t) u\right)(\xi) d t+F_{ \pm} u(\xi) \\
= & -\frac{1}{(2 \pi)^{n / 2}} \lim _{\epsilon \rightarrow 0 \pm}\left\langle G_{-\|\xi\|^{2}}^{\mp}[\gamma] u_{\xi}^{ \pm},\left(\Delta+\|\xi\|^{2}\right)\left(\Delta+\|\xi\|^{2}+i \epsilon\right)^{-1} u\right\rangle+F_{ \pm} u(\xi) \\
= & -\frac{1}{(2 \pi)^{n / 2}}\left\langle G_{-\|\xi\|^{2}}^{\mp}[\gamma] u_{\xi}^{ \pm}, u\right\rangle+F_{ \pm} u(\xi) \\
= & F u(\xi)
\end{aligned}
$$

Let us now introduce the scattering operator $S:=W_{+}^{*} W_{-}$, so that, by $W_{ \pm}=F_{ \pm}^{*} F$, one gets $F S F^{*}=F_{+} F_{-}^{*}$. The scattering matrix

$$
S_{k}: L^{2}\left(\mathbb{S}^{n-1}\right) \rightarrow L^{2}\left(\mathbb{S}^{n-1}\right),
$$

where $\mathbb{S}^{n-1}$ denotes the unit sphere in $\mathbb{R}^{n}$, is then defined by the relation

$$
S_{k}(F u)_{k}=(F S u)_{k}, \quad(F u)_{k}(\hat{\xi}):=F u(k \hat{\xi}) .
$$

Therefore

$$
S_{k}\left(F_{-} u\right)_{k}=\left(F_{+} u\right)_{k} .
$$

The next results shows how the kernel (proportional to the scattering amplitude) of the linear operator $1-S_{k}$ can be expressed in terms of the limit Weyl functions $\Theta+\Pi M_{-k^{2}}^{ \pm} \Pi^{\prime}$; here $\mu$ denotes Lebesgue measure on $\mathbb{S}^{n-1}$.

Theorem 5.6. Assume either (3.6) or (3.7) holds, so that $W_{ \pm}$and hence $S_{k}$ exist. Then, for any $k>0$ such that $-k^{2} \in E_{\Pi, \Theta}^{-}$,

$$
S_{k} f(\hat{\xi})=f(\hat{\xi})-\int_{\mathbb{S}^{n-1}} s_{k}\left(\hat{\xi}, \hat{\xi}^{\prime}\right) f\left(\hat{\xi}^{\prime}\right) d \mu\left(\hat{\xi}^{\prime}\right),
$$

where

$$
\begin{aligned}
s_{k}\left(\hat{\xi}, \hat{\xi}^{\prime}\right): & =\frac{i}{4 \pi}\left(\frac{k}{2 \pi}\right)^{n-2}\left\langle\Pi \gamma u_{k \hat{\xi}^{\prime}}^{\circ},\left(\Theta+\Pi M_{-k^{2}}^{-} \Pi^{\prime}\right)^{-1} \Pi \gamma u_{k \hat{\xi}^{\prime}}^{\circ}\right\rangle \\
& =\frac{i}{4 \pi}\left(\frac{k}{2 \pi}\right)^{n-2}\left\langle\left(\Theta+\Pi M_{-k^{2}}^{+} \Pi^{\prime}\right)^{-1} \Pi \gamma u_{k \hat{\xi}^{\prime}}^{\circ}, \Pi \gamma u_{k \hat{\xi}^{\circ}}^{\circ}\right\rangle .
\end{aligned}
$$

In the case (3.6) holds, one also has the equivalent representation

$$
\begin{aligned}
s_{k}\left(\hat{\xi}, \hat{\xi}^{\prime}\right) & =\frac{i}{4 \pi}\left(\frac{k}{2 \pi}\right)^{n-2}\left\langle\Pi \gamma u_{k \hat{\xi}^{\prime}}^{\circ},\left(B_{\Theta}-\Pi \gamma G_{-k^{2}}^{-} \Pi^{\prime}\right)^{-1} \Pi \gamma u_{k \hat{\xi}^{\prime}}^{\circ}\right\rangle \\
& =\frac{i}{4 \pi}\left(\frac{k}{2 \pi}\right)^{n-2}\left\langle\left(B_{\Theta}-\Pi \gamma G_{-k^{2}}^{+} \Pi^{\prime}\right)^{-1} \Pi \gamma u_{k \hat{\xi}^{\prime}}^{\circ}, \Pi \gamma u_{k \hat{\xi}^{\prime}}^{\circ} .\right.
\end{aligned}
$$


Proof. Here we follow the same strategy as in [37] and [5]. By the definition of $S_{k}$ we only need to show that, for any $u \in L_{\alpha}^{2}\left(\mathbb{R}^{n}\right)$, one has

$$
\left(F_{+} u\right)_{k}(\hat{\xi})=\left(F_{-} u\right)_{k}(\hat{\xi})-\int_{\mathbb{S}^{n-1}} s_{k}\left(\hat{\xi}, \hat{\xi}^{\prime}\right)\left(F_{-} u\right)_{k}\left(\hat{\xi}^{\prime}\right) d \mu\left(\hat{\xi}^{\prime}\right) .
$$

Let us define the auxiliary functions

$$
v_{k \hat{\xi}}:=u_{k \hat{\xi}}^{-}-u_{k \hat{\xi}}^{+}-\int_{\mathbb{S}^{n-1}} s_{k}\left(\hat{\xi}, \hat{\xi}^{\prime}\right) u_{k \hat{\xi}^{\prime}}^{-} d \mu\left(\hat{\xi}^{\prime}\right) .
$$

By (5.4),

$$
\begin{aligned}
v_{k \hat{\xi}} & =G_{-k^{2}}^{+}[\gamma] u_{k \hat{\xi}}^{-}-G_{-k^{2}}^{-}[\gamma] u_{k \hat{\xi}}^{+}-\int_{\mathbb{S}^{n-1}} s_{k}\left(\hat{\xi}, \hat{\xi}^{\prime}\right) G_{-k^{2}}^{+}[\gamma] u_{k \hat{\xi}^{\prime}}^{-} d \mu\left(\hat{\xi}^{\prime}\right) \\
& -\frac{i}{4 \pi}\left(\frac{k}{2 \pi}\right)^{n-2} \int_{\mathbb{S}^{n-1}}\left\langle\gamma u_{k \hat{\xi}^{\prime}}^{\circ},[\gamma] u_{k \hat{\xi}^{\prime}}^{+}\right\rangle u_{k \hat{\xi}^{\prime}}^{\circ} d \mu\left(\hat{\xi}^{\prime}\right) .
\end{aligned}
$$

By

$$
\int_{\mathbb{S}^{n-1}} \bar{u}_{k \hat{\xi}}^{\circ}(x) u_{k \hat{\xi}}^{\circ}(y) d \mu(\hat{\xi})=4 \pi i\left(\frac{2 \pi}{k}\right)^{n-2}\left(\mathscr{G}_{-k^{2}}^{-}(x-y)-\mathscr{G}_{-k^{2}}^{+}(x-y)\right)
$$

(see [5, formula (15)]) and by (5.1)-(15.2), one gets

$$
-\frac{i}{4 \pi}\left(\frac{k}{2 \pi}\right)^{n-2} \int_{\mathbb{S}^{n-1}}\left\langle\gamma u_{k \hat{\xi}^{\prime}}^{\circ},[\gamma] u_{k \hat{\xi}^{\prime}}^{+}\right\rangle u_{k \hat{\xi}^{\prime}}^{\circ} d \mu\left(\hat{\xi}^{\prime}\right)=\left(G_{-k^{2}}^{-}-G_{-k^{2}}^{+}\right)[\gamma] u_{k \hat{\xi}}^{+}
$$

and so

$$
v_{k \hat{\xi}}=G_{-k^{2}}^{+}[\gamma] v_{k \hat{\xi}}
$$

Therefore, by 1) in Lemma [5.3, $v_{k \hat{\xi}}$ satisfies the Sommerfeld radiation condition. Since $u_{k \hat{\xi}}^{ \pm} \in \operatorname{ker}\left(\tilde{\Delta}_{\Pi, \Theta}+k^{2}\right)$, one has $v_{k \hat{\xi}} \in \operatorname{ker}\left(\tilde{\Delta}_{\Pi, \Theta}+k^{2}\right)$. Thus, by 2) in Lemma [5.3, $v_{k \hat{\xi}}=0$ and so

$$
u_{k \hat{\xi}}^{+}=u_{k \hat{\xi}}^{-}-\int_{\mathbb{S}^{n-1}} s_{k}\left(\hat{\xi}, \hat{\xi}^{\prime}\right) u_{k \hat{\xi}^{\prime}}^{-} d \mu\left(\hat{\xi}^{\prime}\right) .
$$

Considering the duality product of both the left and right functions with $u \in L_{\alpha}^{2}\left(\mathbb{R}^{n}\right)$, one gets (5.9) and the proof is done.

Remark 5.7. Given $\mu \in(0,+\infty) \cap \rho\left(\Delta_{\Pi, \Theta}\right)$, let $W_{ \pm}^{\mu}$ denote the wave operators for the scattering couple $\left(\left(-\Delta_{\Pi, \Theta}+\mu\right)^{-1},(-\Delta+\mu)^{-1}\right)$. Since both $W_{ \pm}$and $W_{ \pm}^{\mu}$ exist and are complete, by the Birman-Kato invariance principle one gets $W_{ \pm}^{\mu}=W_{ \pm}$. By (3.4) one has

$$
\left(-\Delta_{\Pi, \Theta}+\mu\right)^{-1}-(-\Delta+\mu)^{-1}=G_{\mu} \Pi^{\prime}\left(\Theta+\Pi M_{\mu} \Pi^{\prime}\right)^{-1} \Pi G_{\mu}^{\prime} .
$$

The Birman-Yafaev general scheme in stationary scattering theory (see e.g. [15, 40], 41]), conditional on the existence of the limit operator

$$
B_{\lambda}^{+}:=\lim _{\epsilon \downarrow 0} B_{\lambda+i \epsilon}, \quad B_{z}:=\Pi G_{\mu}^{\prime}\left((-\Delta+\mu)^{-1}-z\right)^{-1} G_{\mu} \Pi^{\prime}
$$


and of the inverse $\left(1+B_{\lambda}^{+}\left(\Theta+\Pi M_{\mu} \Pi^{\prime}\right)^{-1}\right)^{-1}$, allows the representation formula for the scattering matrix $S_{\lambda}^{\mu}$, corresponding to the scattering operator $S^{\mu}=\left(W_{+}^{\mu}\right)^{*} W_{-}^{\mu}$, given by (see e.g. [41, equation (2.8)])

$$
S_{\lambda}^{\mu}=1-2 \pi i L_{\lambda} \Pi^{\prime}\left(\Theta+\Pi M_{\mu} \Pi^{\prime}\right)^{-1}\left(1+B_{\lambda}^{+}\left(\Theta+\Pi M_{\mu} \Pi^{\prime}\right)^{-1}\right)^{-1} \Pi L_{\lambda}^{\prime} .
$$

Here

$$
\begin{aligned}
& L_{\lambda}: H^{-3 / 2}(\Gamma) \oplus H^{-1 / 2}(\Gamma) \rightarrow L^{2}\left(\mathbb{S}^{n-1}\right), \\
& \left(L_{\lambda}(\phi \oplus \varphi)\right)(\hat{\xi}):=\left[\left(F_{0} G_{\mu}(\phi \oplus \varphi)\right)(\lambda)\right](\hat{\xi})
\end{aligned}
$$

is defined in terms of the unitary map $F_{0}: L^{2}\left(\mathbb{R}^{n}\right) \rightarrow L^{2}\left(\left(0, \mu^{-1}\right) ; \mathbb{S}^{n-1}\right)$ such that the operator $F_{0}(-\Delta+\mu)^{-1} F_{0}^{*}$ acts as multiplication by $\lambda$, i.e.

$$
\left[\left(F_{0} u\right)(\lambda)\right](\hat{\xi}):=2^{-\frac{1}{2}}\left(\frac{1}{\lambda}-\mu\right)^{\frac{n-2}{4}}(F u)\left(\left(\frac{1}{\lambda}-\mu\right)^{\frac{1}{2}} \hat{\xi}\right) .
$$

By the identities

$$
\begin{gathered}
\left((-\Delta+\mu)^{-1}-z\right)^{-1}=-\frac{1}{z}\left(1+\frac{1}{z}\left(-\Delta+\mu-\frac{1}{z}\right)^{-1}\right) \\
G_{\mu}^{\prime}\left(-\Delta+\mu-\frac{1}{z}\right)^{-1}=z\left(G_{\mu-\frac{1}{z}}^{\prime}-G_{\mu}^{\prime}\right) \\
M_{\mu-\frac{1}{z}}=M_{\mu}-\frac{1}{z} G_{\mu-\frac{1}{z}}^{\prime} G_{\mu}
\end{gathered}
$$

one obtains

$$
\begin{aligned}
& \left(\Theta+\Pi M_{\mu} \Pi^{\prime}\right)^{-1}\left(1+B_{z}\left(\Theta+\Pi M_{\mu} \Pi^{\prime}\right)^{-1}\right)^{-1}=\left(\Theta+\Pi M_{\mu} \Pi^{\prime}-B_{z}\right)^{-1} \\
= & \left(\Theta+\Pi M_{\mu} \Pi^{\prime}-\Pi G_{\mu}^{\prime}\left(\frac{1}{z}+\frac{1}{z^{2}}\left(-\Delta+\mu-\frac{1}{z}\right)^{-1}\right) G_{\mu} \Pi^{\prime}\right)^{-1} \\
= & \left(\Theta+\Pi M_{\mu} \Pi^{\prime}-\frac{1}{z} \Pi G_{\mu}^{\prime} G_{\mu} \Pi^{\prime}-\frac{1}{z} \Pi\left(G_{\mu-\frac{1}{z}}^{\prime}-G_{\mu}^{\prime}\right) G_{\mu} \Pi^{\prime}\right)^{-1} \\
= & \left(\Theta+\Pi M_{\mu} \Pi^{\prime}-\frac{1}{z} \Pi G_{\mu-\frac{1}{z}}^{\prime} G_{\mu} \Pi^{\prime}\right)^{-1}=\left(\Theta+\Pi M_{\mu-\frac{1}{z}} \Pi^{\prime}\right)^{-1} .
\end{aligned}
$$

Therefore, by Theorems 4.2 and 4.5 , both $B_{\lambda}^{+}$and $\left(1+B_{\lambda}^{+}\left(\Theta+\Pi M_{\mu} \Pi^{\prime}\right)^{-1}\right)^{-1}$ are well defined and

$$
S_{\lambda}^{\mu}=1-2 \pi i L_{\lambda} \Pi^{\prime}\left(\Theta+\Pi M_{\mu-\frac{1}{\lambda}}^{+} \Pi^{\prime}\right)^{-1} \Pi L_{\lambda}^{\prime}, \quad \mu-\frac{1}{\lambda} \in E_{\Pi, \Theta}^{-} .
$$

In case Theorems 4.2 and 4.5 were not available, using the results contained in [40, Chapter 7, Sections 4 and 6], the representation formula (5.11) could be still obtained under Kato-smoothness or trace-class hypotheses on the resolvent difference (5.10). However for the models we are here considering, the trace-class condition is not always fulfilled while checking the 
smoothness property may be a substantial problem (see e.g. [40, Chapter 7, Sections 4, Proposition 1]).

Finally, using the correspondence $S_{\lambda}^{\mu}=S_{k}$, which holds whenever $\mu-\frac{1}{\lambda}=$ $-k^{2}$ (see [40, Section 6, Chapter 2]), and the identity

$$
\left(L_{\lambda}(\phi \oplus \varphi)\right)(\hat{\xi})=2^{-\frac{1}{2}}\left(\frac{1}{\lambda}-\mu\right)^{\frac{n-2}{4}}(2 \pi)^{-\frac{n}{2}}\left\langle\gamma u_{\left(\lambda^{-1}-\mu\right)^{\frac{1}{2}} \hat{\xi}}^{\circ}, \phi \oplus \varphi\right\rangle,
$$

one gets that (5.12) matches the formula provided in Theorem 5.6.

\section{EXAMPles: Boundary CONDITIONS ON $\Gamma$}

In this section we apply our results to self-adjoint realizations of the Laplacian with various kind of boundary conditions on $\Gamma$. For more details on such models we refer to [29, Section 5]. In particular, by the results given there, hypothesis (3.6) holds for all the examples presented here. As regards the semi-boundedness hypotheses required in Theorem 4.2, the semi-boundedness of the operators $\Delta_{D}$ and $\Delta_{N}$ in subsections 6.1 and 6.2 is clear, semi-boundedness of $\Delta_{R}$ in subsection 6.3] is provided in [29, Remark 5.2] and semiboundedness of $\Delta_{\alpha, \delta}$ and $\Delta_{\beta, \delta^{\prime}}$ in subsections 6.4 and 6.5 is provided in [9, Theorem 3.16] (see also the next Section, the proofs being essentially the same). In the following, in order to simplify the exposition, we suppose that $\Omega_{\mathrm{ex}}$ is connected.

6.1. Dirichlet boundary conditions. Let us consider the self-adjoint extension $\Delta_{D}$ corresponding to Dirichlet boundary conditions on the whole $\Gamma$; it is given by the direct sum $\Delta_{D}=\Delta_{\text {in }}^{D} \oplus \Delta_{\text {ex }}^{D}$, where the self-adjoint operators $\Delta_{\text {in }}^{D}$ and $\Delta_{\text {ex }}^{D}$ are defined by $\Delta_{\text {in }}^{D}:=\Delta \mid \operatorname{dom}\left(\Delta_{\text {in }}^{D}\right)$ and $\Delta_{\text {ex }}^{D}:=$ $\Delta \mid \operatorname{dom}\left(\Delta_{\text {ex }}^{D}\right)$, with domains $\operatorname{dom}\left(\Delta_{\text {in }}^{D}\right)=\left\{u_{\text {in }} \in H^{2}\left(\Omega_{\text {in }}\right): \gamma_{0}^{\text {in }} u_{\text {in }}=0\right\}$ and $\operatorname{dom}\left(\Delta_{\mathrm{ex}}^{D}\right)=\left\{u_{\mathrm{ex}} \in H^{2}\left(\Omega_{\mathrm{ex}}\right): \gamma_{0}^{\mathrm{ex}} u_{\mathrm{ex}}=0\right\}$. Since

$$
\begin{aligned}
\operatorname{dom}\left(\Delta_{\text {in }}^{D}\right) \oplus \operatorname{dom}\left(\Delta_{\text {ex }}^{D}\right) & =\left\{u \in H^{2}\left(\mathbb{R}^{n} \backslash \Gamma\right):\left[\gamma_{0}\right] u=0, \gamma_{0} u=0\right\} \\
& =\left\{u \in H^{1}\left(\mathbb{R}^{n}\right) \cap H^{2}\left(\mathbb{R}^{n} \backslash \Gamma\right): \gamma_{0} u=0\right\},
\end{aligned}
$$

that corresponds, in Corollary [3.6, to the choice $\Pi(\phi \oplus \varphi):=\phi \oplus 0$, and $B_{\Theta}=0$. Thus (see [29, Subsection 5.1])

$$
\left(\Delta_{\text {in }}^{D} \oplus \Delta_{\text {ex }}^{D}\right) u=\Delta u-\left[\gamma_{1}\right] u \delta_{\Gamma}
$$

and, by $\left(\gamma_{0} S L_{z}\right)^{-1}=P_{z}^{\text {in }}-P_{z}^{\text {ex }}$, where $P_{z}^{\text {in }}$ and $P_{z}^{\text {ex }}$ denote the Dirichletto-Neumann operators for $\Omega_{\text {in }}$ and $\Omega_{\text {ex }}$ respectively (see e.g. [29, equation (5.4)]), one has, for any $z \in \mathbb{C} \backslash(-\infty, 0]$,

$$
\left(-\left(\Delta_{\text {in }}^{D} \oplus \Delta_{\text {ex }}^{D}\right)+z\right)^{-1}=(-\Delta+z)^{-1}+S L_{z}\left(P_{z}^{\text {ex }}-P_{z}^{\text {in }}\right) \gamma_{0}(-\Delta+z)^{-1} .
$$


Then, by Theorem [5.6, one has, for any $k>0$ such that $-k^{2} \notin \sigma\left(\Delta_{\text {in }}^{D}\right)$,

$$
s_{k}\left(\hat{\xi}, \hat{\xi}^{\prime}\right)=\frac{i}{4 \pi}\left(\frac{k}{2 \pi}\right)^{n-2}\left\langle\left(P_{-k^{2}}^{\mathrm{ex}}-P_{-k^{2}}^{\text {in }}\right)^{+} \gamma_{0} u_{k \hat{\xi}^{\prime}}^{\circ}, \gamma_{0} u_{k \hat{\xi}^{\prime}}^{\circ}\right\rangle
$$

where

$$
\left(P_{-k^{2}}^{\mathrm{ex}}-P_{-k^{2}}^{\mathrm{in}}\right)^{+}:=\lim _{\epsilon \downarrow 0}\left(P_{-k^{2}+i \epsilon}^{\mathrm{ex}}-P_{-k^{2}+i \epsilon}^{\mathrm{in}}\right)=-\left(\gamma_{0} S L_{-k^{2}}^{+}\right)^{-1} .
$$

Such a limit exists in $\mathrm{B}\left(H^{\frac{3}{2}}(\Gamma), H^{-\frac{3}{2}}(\Gamma)\right)$ by Theorem 4.5. Notice that, restricted to the case $n=2$, similar formulae have been obtained (without the smoothness condition on $\Gamma$ ) in [19, Theorems 5.3 and 5.6].

6.2. Neumann boundary conditions. Let us consider the self-adjoint extension $\Delta^{N}$ corresponding to Neumann boundary conditions on the whole $\Gamma$; it is given by the direct sum $\Delta_{N}=\Delta_{\text {in }}^{N} \oplus \Delta_{\text {ex }}^{N}$, where the self-adjoint operators $\Delta_{\text {in }}^{N}$ and $\Delta_{\text {ex }}^{N}$ are defined by $\Delta_{\text {in }}^{N}:=\Delta \mid \operatorname{dom}\left(\Delta_{\text {in }}^{N}\right)$ and $\Delta_{\text {ex }}^{N}:=$ $\Delta \mid \operatorname{dom}\left(\Delta_{\text {ex }}^{N}\right)$, with domains $\operatorname{dom}\left(\Delta_{\text {in }}^{N}\right)=\left\{u_{\text {in }} \in H^{2}\left(\Omega_{\text {in }}\right): \gamma_{1}^{\text {in }} u_{\text {in }}=0\right\}$ and $\operatorname{dom}\left(\Delta_{\mathrm{ex}}^{N}\right)=\left\{u_{\mathrm{ex}} \in H^{2}\left(\Omega_{\mathrm{ex}}\right): \gamma_{1}^{\mathrm{ex}} u_{\mathrm{ex}}=0\right\}$. Since

$$
\operatorname{dom}\left(\Delta_{\mathrm{in}}^{N}\right) \oplus \operatorname{dom}\left(\Delta_{\mathrm{ex}}^{N}\right)=\left\{u \in H^{2}\left(\mathbb{R}^{n} \backslash \Gamma\right):\left[\gamma_{1}\right] u=\gamma_{1} u=0\right\},
$$

that corresponds, in Corollary [3.6, to the choice $\Pi(\phi \oplus \varphi):=0 \oplus \varphi$, and $B_{\Theta}=0$. Thus (see [29, Subsection 5.2])

$$
\left(\Delta_{\mathrm{in}}^{N} \oplus \Delta_{\mathrm{ex}}^{N}\right) u=\Delta u-\left[\gamma_{0}\right] u \nu \cdot \nabla \delta_{\Gamma},
$$

and, by $\left(\gamma_{1} D L_{z}\right)^{-1}=Q_{z}^{\text {ex }}-Q_{z}^{\text {in }}$, where $Q_{z}^{\text {in }}$ and $Q_{z}^{\text {ex }}$ denote the Neumannto-Dirichlet operators for $\Omega_{\mathrm{in}}$ and $\Omega_{\mathrm{ex}}$ respectively (see e.g. [29, equation (5.7)]), one has, for any $z \in \mathbb{C} \backslash(-\infty, 0]$,

$$
\left(-\left(\Delta_{\mathrm{in}}^{N} \oplus \Delta_{\mathrm{ex}}^{N}\right)+z\right)^{-1}=(-\Delta+z)^{-1}+D L_{z}\left(Q_{z}^{\mathrm{in}}-Q_{z}^{\mathrm{ex}}\right) \gamma_{1}(-\Delta+z)^{-1} .
$$

Then, by Theorem 5.6, one has, for any $k>0$ such that $-k^{2} \notin \sigma\left(\Delta_{\text {in }}^{N}\right)$,

$$
s_{k}\left(\hat{\xi}, \hat{\xi}^{\prime}\right)=\frac{i}{4 \pi}\left(\frac{k}{2 \pi}\right)^{n-2}\left\langle\left(Q_{-k^{2}}^{\text {in }}-Q_{-k^{2}}^{\text {ex }}\right)^{+} \gamma_{1} u_{k \hat{\xi}^{\prime}}^{\circ}, \gamma_{1} u_{k \hat{\xi}^{\circ}}^{\circ},\right.
$$

where

$$
\left(Q_{-k^{2}}^{\mathrm{in}}-Q_{-k^{2}}^{\mathrm{ex}}\right)^{+}:=\lim _{\epsilon \downarrow 0}\left(Q_{-k^{2}+i \epsilon}^{\mathrm{in}}-Q_{-k^{2}+i \epsilon}^{\mathrm{ex}}\right)=-\left(\gamma_{1} D L_{-k^{2}}^{+}\right)^{-1} .
$$

Such a limits exists in $\mathrm{B}\left(H^{\frac{1}{2}}(\Gamma), H^{-\frac{1}{2}}(\Gamma)\right)$ by Theorem 4.5. Notice that, restricted to the case $n=2$, similar formulae have been obtained (without the smoothness condition on $\Gamma$ ) in [21, Theorems 4.2 and 4.3]. 
6.3. Robin boundary conditions. Let us consider the self-adjoint extension $\Delta_{R}$ corresponding to Robin boundary conditions on the whole $\Gamma$; it is given by the direct $\operatorname{sum} \Delta_{R}=\Delta_{\text {in }}^{R} \oplus \Delta_{\mathrm{ex}}^{R}$, where

$$
\begin{gathered}
\Delta_{\text {in }}^{R}:=\Delta\left|\operatorname{dom}\left(A_{\mathrm{in}}^{R}\right), \quad \Delta_{\mathrm{ex}}^{R}:=\Delta\right| \operatorname{dom}\left(\Delta_{\mathrm{ex}}^{R}\right), \\
\operatorname{dom}\left(\Delta_{\mathrm{in}}^{R}\right)=\left\{u_{\mathrm{in}} \in \operatorname{dom}\left(\Delta_{\mathrm{in}}^{\max }\right): \gamma_{1}^{\mathrm{in}} u_{\text {in }}=b_{\text {in }} \gamma_{0}^{\mathrm{in}} u_{\mathrm{in}}\right\}, \\
\operatorname{dom}\left(\Delta_{\mathrm{ex}}^{R}\right)=\left\{u_{\mathrm{ex}} \in \operatorname{dom}\left(\Delta_{\mathrm{ex}}^{\max }\right): \gamma_{1}^{\mathrm{ex}} u_{\mathrm{ex}}=b_{\mathrm{ex}} \gamma_{0}^{\mathrm{ex}} u_{\mathrm{ex}}\right\} .
\end{gathered}
$$

Here $b_{\text {in }}$ and $b_{\text {ex }}$ are real-valued multipliers in $H^{\frac{1}{2}}(\Gamma)$. Since, in case $b_{\text {ex }}(x) \neq$ $b_{\text {in }}(x)$ for a.e. $x \in \Gamma$, the domain of $\Delta_{\text {in }}^{R} \oplus \Delta_{\mathrm{ex}}^{R}$ is given by

$$
\begin{aligned}
& \operatorname{dom}\left(\Delta_{\mathrm{in}}^{R} \oplus \Delta_{\mathrm{ex}}^{R}\right) \\
&=\left\{u \in H^{2}\left(\mathbb{R}^{n} \backslash \Gamma\right):\left(b_{\mathrm{ex}}-b_{\mathrm{in}}\right) \gamma_{0} u=\left[\gamma_{1}\right] u-\frac{1}{2}\left(b_{\mathrm{ex}}+b_{\mathrm{in}}\right)\left[\gamma_{0}\right] u,\right. \\
&\left.\left(b_{\mathrm{ex}}-b_{\mathrm{in}}\right) \gamma_{1} u=\frac{1}{2}\left(b_{\mathrm{ex}}+b_{\mathrm{in}}\right)\left[\gamma_{1}\right] u-b_{\mathrm{ex}} b_{\mathrm{in}}\left[\gamma_{0}\right] u\right\} .
\end{aligned}
$$

that corresponds, in Corollary [3.6, to the choice $\Pi=1$ and $B_{\Theta}=B_{R}$, where

$$
B_{R}=-\frac{1}{[b]}\left[\begin{array}{cc}
1 & \langle b\rangle \\
\langle b\rangle & b_{\mathrm{ex}} b_{\mathrm{in}}
\end{array}\right], \quad\langle b\rangle:=\frac{1}{2}\left(b_{\mathrm{ex}}+b_{\mathrm{in}}\right), \quad[b]:=b_{\mathrm{ex}}-b_{\mathrm{in}},
$$

Thus (see [29, Subsection 5.3])

$$
\left(\Delta_{\text {in }}^{R} \oplus \Delta_{\text {ex }}^{R}\right) u=\Delta u-\frac{4}{[b]}\left(\left(\langle b\rangle \gamma_{1} u-b_{\text {ex }} b_{\text {in }} \gamma_{0} u\right) \delta_{\Gamma}+\left(\gamma_{1} u-\langle b\rangle \gamma_{0} u\right) \nu \cdot \nabla \delta_{\Gamma}\right)
$$

and, for any $z \in \rho\left(\Delta_{\text {in }}^{R}\right) \cap \rho\left(\Delta_{\text {ex }}^{R}\right) \cap \mathbb{C} \backslash(-\infty, 0]$,

$$
\begin{aligned}
& \left(-\left(\Delta_{\text {in }}^{R} \oplus \Delta_{\text {ex }}^{R}\right)+z\right)^{-1} \\
= & (-\Delta+z)^{-1}-G_{z}\left[\begin{array}{cc}
1 /[b]+\gamma_{0} S L_{z} & \langle b\rangle /[b]+\gamma_{0} D L_{z} \\
\langle b\rangle /[b]+\gamma_{1} S L_{z} & b_{+} b_{-} /[b]+\gamma_{1} D L_{z}
\end{array}\right]^{-1} \gamma(-\Delta+z)^{-1},
\end{aligned}
$$

where $G_{z}(\phi \oplus \varphi)=S L_{z} \phi+D L_{z} \varphi$. Let us notice that the case in which one has the same Robin boundary conditions on both sides of $\Gamma$ corresponds to the choice $b_{\mathrm{ex}}=b_{\circ}=-b_{\text {in }}$.

Then, by Theorem 5.6, one has, for any $k>0$ such that $-k^{2} \notin \sigma\left(\Delta_{\text {in }}^{R}\right)$,

$$
\begin{aligned}
& s_{k}\left(\hat{\xi}, \hat{\xi}^{\prime}\right) \\
= & -\frac{i}{4 \pi}\left(\frac{k}{2 \pi}\right)^{n-2}\left\langle\left[\begin{array}{cc}
1 /[b]+\gamma_{0} S L_{-k^{2}}^{+} & \langle b\rangle /[b]+\gamma_{0} D L_{-k^{2}}^{+} \\
\langle b\rangle /[b]+\gamma_{1} S L_{-k^{2}}^{+} & b_{+} b_{-} /[b]+\gamma_{1} D L_{-k^{2}}^{+}
\end{array}\right]^{-1} \gamma u_{k \xi^{\prime}}^{\circ}, \gamma u_{k \xi}^{\circ}\right\rangle .
\end{aligned}
$$


6.4. $\delta$-interactions. Here we consider the self-adjoint extension corresponding to the choice $\Pi(\phi \oplus \varphi)=\phi \oplus 0$ and $\Theta(\phi \oplus \varphi)=-\left(\phi / \alpha+\gamma_{0} S L \phi\right) \oplus 0$, where $\alpha$ is a real-valued multiplier in $H^{\frac{3}{2}}(\Gamma)$ such that $1 / \alpha \in L^{\infty}(\Gamma)$. Such a kind of self-adjoint extensions correspond to the boundary conditions $\alpha \gamma_{0} u=\left[\gamma_{1}\right] u$ and so one obtains the self-adjoint extensions usually called " $\delta$-interactions on $\Gamma "$ (see [16], 9] and references therein). By Corollary 3.6 (see [29, Subsection 5.4]), one gets the self-adjoint extension

$$
\begin{gathered}
\Delta_{\alpha, \delta} u=\Delta u-\alpha \gamma_{0} u \delta_{\Gamma}, \\
\operatorname{dom}\left(\Delta_{\alpha, \delta}\right):=\left\{u \in H^{1}\left(\mathbb{R}^{n}\right) \cap H^{2}\left(\mathbb{R}^{n} \backslash \Gamma\right): \alpha \gamma_{0} u=\left[\gamma_{1}\right] u\right\} ;
\end{gathered}
$$

its resolvent is given by

$$
\begin{aligned}
\left(-\Delta_{\alpha, \delta}+z\right)^{-1} & =(-\Delta+z)^{-1}-S L_{z}\left((1 / \alpha)+\gamma_{0} S L_{z}\right)^{-1} \gamma_{0}(-\Delta+z)^{-1} \\
& =(-\Delta+z)^{-1}-S L_{z}\left(1+\alpha \gamma_{0} S L_{z}\right)^{-1} \alpha \gamma_{0}(-\Delta+z)^{-1} .
\end{aligned}
$$

Then, by Theorem 5.6 and Remark 3.8, one has, for any $k>0$,

$$
s_{k}\left(\hat{\xi}, \hat{\xi}^{\prime}\right)=-\frac{i}{4 \pi}\left(\frac{k}{2 \pi}\right)^{n-2}\left\langle\left(1+\alpha \gamma_{0} S L_{-k^{2}}^{+}\right)^{-1} \alpha \gamma_{0} u_{k \hat{\xi}^{\prime}}^{\circ}, \gamma_{0} u_{k \hat{\xi}}^{\circ}\right\rangle .
$$

6.5. $\delta^{\prime}$-interactions. Here we consider the self-adjoint extension corresponding to the choice $\Pi(\phi \oplus \varphi)=0 \oplus \varphi$ and $\Theta(\phi \oplus \varphi)=0 \oplus\left(\varphi / \beta-\gamma_{1} D L \varphi\right)$, where $\beta$ is a real-valued multiplier in $H^{\frac{1}{2}}(\Gamma)$ such that $1 / \beta \in L^{\infty}(\Gamma)$. Such a kind of self-adjoint extensions correspond to the boundary conditions $\beta \gamma_{1} u=\left[\gamma_{0}\right] u$ and so one obtains the self-adjoint extensions usually called " $\delta$-interactions on $\Gamma$ " (see [9] and references therein). By Corollary 3.6 (see [29, Subsection 5.5]), one gets the self-adjoint extension

$$
\begin{gathered}
\Delta_{\beta, \delta^{\prime}} u=\Delta u-\beta \gamma_{1} u \nu \cdot \nabla \delta_{\Gamma}, \\
\operatorname{dom}\left(\Delta_{\beta, \delta^{\prime}}\right):=\left\{u \in H^{2}\left(\mathbb{R}^{n} \backslash \Gamma\right):\left[\gamma_{1}\right] u=0, \beta \gamma_{1} u=\left[\gamma_{0}\right] u\right\} ;
\end{gathered}
$$

Its resolvent is given by

$$
\begin{aligned}
\left(-\Delta_{\beta, \delta^{\prime}}+z\right)^{-1} & =(-\Delta+z)^{-1}+D L_{z}\left((1 / \beta)-\gamma_{1} D L_{z}\right)^{-1} \gamma_{1}(-\Delta+z)^{-1} \\
& =(-\Delta+z)^{-1}+D L_{z}\left(1-\beta \gamma_{1} D L_{z}\right)^{-1} \beta \gamma_{1}(-\Delta+z)^{-1} .
\end{aligned}
$$

Then, by Theorem 5.6 and Remark 3.8, one has, for any $k>0$,

$$
s_{k}\left(\hat{\xi}, \hat{\xi}^{\prime}\right)=\frac{i}{4 \pi}\left(\frac{k}{2 \pi}\right)^{n-2}\left\langle\left(1-\beta \gamma_{1} D L_{-k^{2}}^{+}\right)^{-1} \beta \gamma_{1} u_{k \hat{\xi}^{\prime}}^{\circ}, \gamma_{1} u_{k \hat{\xi}}^{\circ}\right\rangle .
$$




\section{EXAMPles: BOUNDARY CONDITIONS ON $\Sigma \subset \Gamma$}

In this section we consider boundary conditions supported on a relatively open part $\Sigma \subset \Gamma$ with Lipschitz boundary. For more details and proof regarding such models we refer to [29, Section 6]. In particular, by the results given there, hypothesis (3.7) holds for all the examples presented here; moreover, the semi-boundedness hypothesis required in Theorem 4.2 holds true as well: this point is next discussed case-by-case. In order to apply Theorem 3.7, so to simplify the exposition, we suppose that $\mathbb{R}^{n} \backslash \bar{\Sigma}$ is connected.

In the following, given $X \subset \Gamma$ closed, we use the definition

$$
H_{X}^{s}(\Gamma):=\left\{\phi \in H^{s}(\Gamma): \operatorname{supp}(\phi) \subseteq X\right\} .
$$

Given $\Sigma \subset \Gamma$ relatively open of class $\mathcal{C}^{0,1}$, we denote by $\Pi_{\Sigma}$ the orthogonal projector in the Hilbert space $H^{s}(\Gamma), s>0$, such that $\operatorname{ran}\left(\Pi_{\Sigma}\right)=H_{\Sigma^{c}}^{s}(\Gamma)^{\perp}$. One has $\operatorname{ran}\left(\Pi_{\Sigma}^{\prime}\right)=H_{\bar{\Sigma}}^{-s}(\Gamma)$, where $\Pi_{\Sigma}^{\prime}=\Lambda^{2 s} \Pi_{\Sigma} \Lambda^{-2 s}$ is the dual projection. In the following, we use the identifications $H_{\Sigma^{c}}^{s}(\Gamma)^{\perp} \simeq H^{s}(\Sigma)$ and $H_{\bar{\Sigma}}^{-s}(\Gamma) \simeq H^{s}(\Sigma)^{\prime}$. In particular, by the former, the orthogonal projection $\Pi_{\Sigma}$ can be identified with the restriction map $R_{\Sigma}: H^{s}(\Gamma) \rightarrow H^{s}(\Sigma)$, $R_{\Sigma} \phi:=\phi \mid \Sigma$.

7.1. Dirichlet boundary conditions. We denote by $\Delta_{D, \Sigma}$ the self-adjoint extension corresponding to the orthogonal projector defined by $\Pi(\phi \oplus \varphi):=$ $\left(\Pi_{\Sigma} \phi\right) \oplus 0 \equiv(\phi \mid \Sigma) \oplus 0$ and to the self-adjoint operator $\Theta(\phi \oplus \varphi):=$ $\left(-\Theta_{D, \Sigma} \phi\right) \oplus 0$

$$
\begin{gathered}
\Theta_{D, \Sigma}: \operatorname{dom}\left(\Theta_{D, \Sigma}\right) \subseteq H_{\bar{\Sigma}}^{-\frac{3}{2}}(\Gamma) \rightarrow H^{\frac{3}{2}}(\Sigma), \quad \Theta_{D, \Sigma} \phi:=\left(\gamma_{0} S L \phi\right) \mid \Sigma, \\
\operatorname{dom}\left(\Theta_{D, \Sigma}\right):=\left\{\phi \in H_{\bar{\Sigma}}^{-\frac{1}{2}}(\Gamma):\left(\gamma_{0} S L \phi\right) \mid \Sigma \in H^{\frac{3}{2}}(\Sigma)\right\} .
\end{gathered}
$$

By Theorem 3.1 (see [29, Subsection 6.1]),

$$
\Delta_{D, \Sigma} u=\Delta u-\left[\hat{\gamma}_{1}\right] u \delta_{\bar{\Sigma}},
$$

$$
\begin{aligned}
& \operatorname{dom}\left(\Delta_{D, \Sigma}\right) \\
= & \left\{u \in H^{1}\left(\mathbb{R}^{n}\right) \cap H_{\Delta}^{0}\left(\mathbb{R}^{n} \backslash \Gamma\right):\left[\hat{\gamma}_{1}\right] u \in \operatorname{dom}\left(\Theta_{D, \Sigma}\right),\left(\gamma_{0}^{\text {in }} u\right)\left|\Sigma=\left(\gamma_{0}^{\text {ex }} u\right)\right| \Sigma=0\right\}
\end{aligned}
$$

is self-adjoint and

$$
\left(-\Delta_{D, \Sigma}+z\right)^{-1}=(-\Delta+z)^{-1}-S L_{z} \Pi_{\Sigma}^{\prime}\left(R_{\Sigma} \gamma_{0} S L_{z} \Pi_{\Sigma}^{\prime}\right)^{-1} R_{\Sigma} \gamma_{0}(-\Delta+z)^{-1} .
$$

Denoting by $\langle\cdot, \cdot\rangle_{-1,1}$ the $H^{-1}\left(\mathbb{R}^{n}\right)-H^{1}\left(\mathbb{R}^{n}\right)$ duality, for any $u \in \operatorname{dom}\left(\Delta_{D, \Sigma}\right) \subset$ $H^{1}\left(\mathbb{R}^{n}\right)$ one has, by (17.1) and $\left\langle\delta_{\Sigma}, u\right\rangle_{-1,1}=0$ whenever $\operatorname{supp}\left(\gamma_{0} u\right) \subseteq \Sigma^{c}$,

$$
\left\langle-\Delta_{D, \Sigma} u, u\right\rangle_{L^{2}\left(\mathbb{R}^{n}\right)}=\langle-\Delta u, u\rangle_{-1,1}=\|\nabla u\|_{L^{2}\left(\mathbb{R}^{n}\right)}^{2}
$$

and so $\Delta_{D, \Sigma} \leq 0$. 
By Theorems 5.6 and 3.7, one gets, for any $k>0$,

$$
s_{k}\left(\hat{\xi}, \hat{\xi}^{\prime}\right)=-\frac{i}{4 \pi}\left(\frac{k}{2 \pi}\right)^{n-2}\left\langle\left(R_{\Sigma} \gamma_{0} S L_{-k^{2}}^{+} \Pi_{\Sigma}^{\prime}\right)^{-1} R_{\Sigma} \gamma_{0} u_{k \hat{\xi}^{\prime}}^{\circ}, R_{\Sigma} \gamma_{0} u_{k \hat{\xi}^{\circ}}^{\circ}\right\rangle
$$

7.2. Neumann boundary conditions. We denote by $\Delta_{N, \Sigma}$ the self-adjoint extension corresponding to the orthogonal projector defined by $\Pi(\phi \oplus \varphi):=$ $0 \oplus\left(\Pi_{\Sigma} \varphi\right) \equiv 0 \oplus(\varphi \mid \Sigma)$ and to the self-adjoint operator $\Theta(\phi \oplus \varphi):=$ $0 \oplus\left(-\Theta_{N, \Sigma} \varphi\right)$,

$$
\begin{gathered}
\Theta_{N, \Sigma}: \operatorname{dom}\left(\Theta_{N, \Sigma}\right) \subseteq H_{\bar{\Sigma}}^{-\frac{1}{2}}(\Gamma) \rightarrow H^{\frac{1}{2}}(\Sigma), \quad \Theta_{N, \Sigma} \varphi=\left(\gamma_{1} D L \varphi\right) \mid \Sigma \\
\operatorname{dom}\left(\Theta_{N, \Sigma}\right):=\left\{\varphi \in H_{\bar{\Sigma}}^{\frac{1}{2}}(\Gamma):\left(\gamma_{1} D L \varphi\right) \mid \Sigma \in H^{\frac{1}{2}}(\Sigma)\right\}
\end{gathered}
$$

By Theorem 3.1 (see [29, Subsection 6.2]),

$$
\Delta_{N, \Sigma} u=\Delta u-\left[\gamma_{0}\right] u \nu \cdot \nabla \delta_{\bar{\Sigma}}
$$

$$
\begin{gathered}
\operatorname{dom}\left(\Delta_{N, \Sigma}\right)=\left\{u \in H^{1}\left(\mathbb{R}^{n} \backslash \bar{\Sigma}\right) \cap H_{\Delta}^{0}\left(\mathbb{R}^{n} \backslash \Gamma\right):\left[\gamma_{0}\right] u \in \operatorname{dom}\left(\Theta_{N, \Sigma}\right),\right. \\
\left.\left[\hat{\gamma}_{1}\right] u=0,\left(\hat{\gamma}_{1}^{\text {in }} u\right)\left|\Sigma=\left(\hat{\gamma}_{1}^{\mathrm{ex}} u\right)\right| \Sigma=0\right\}
\end{gathered}
$$

is self-adjoint and

$$
\begin{aligned}
& \left(-\Delta_{N, \Sigma}+z\right)^{-1} \\
= & (-\Delta+z)^{-1}-D L_{z} \Pi_{\Sigma}^{\prime}\left(R_{\Sigma} \hat{\gamma}_{1} D L_{z} \Pi_{\Sigma}^{\prime}\right)^{-1} R_{\Sigma} \gamma_{1}(-\Delta+z)^{-1} .
\end{aligned}
$$

By Green's formula (2.8), for any $u \in \operatorname{dom}\left(\Delta_{N, \Sigma}\right) \subset H^{1}\left(\mathbb{R}^{n} \backslash \Gamma\right) \cap H_{\Delta}^{0}\left(\mathbb{R}^{n} \backslash \Gamma\right)$ one has

$$
\begin{aligned}
\left\langle-\Delta_{N, \Sigma} u, u\right\rangle_{L^{2}\left(\mathbb{R}^{n}\right)} & =\|\nabla u\|_{L^{2}\left(\Omega_{\mathrm{in}}\right)}^{2}+\|\nabla u\|_{L^{2}\left(\Omega_{\mathrm{ex}}\right)}^{2}+\left\langle\hat{\gamma}_{1} u,\left[\gamma_{0}\right] u\right\rangle_{L^{2}(\Gamma)} \\
& =\|\nabla u\|_{L^{2}\left(\Omega_{\mathrm{in}}\right)}^{2}+\|\nabla u\|_{L^{2}\left(\Omega_{\mathrm{ex}}\right)}^{2}
\end{aligned}
$$

and so $\Delta_{N, \Sigma} \leq 0$.

Then, by Theorems 5.6 and 3.7, one gets, for any $k>0$,

$$
s_{k}\left(\hat{\xi}, \hat{\xi}^{\prime}\right)=-\frac{i}{4 \pi}\left(\frac{k}{2 \pi}\right)^{n-2}\left\langle\left(R_{\Sigma} \hat{\gamma}_{1} D L_{-k^{2}}^{+} \Pi_{\Sigma}^{\prime}\right)^{-1} R_{\Sigma} \gamma_{1} u_{k \hat{\xi}^{\prime}}^{\circ}, R_{\Sigma} \gamma_{1} u_{k \hat{\xi}}^{\circ}\right\rangle .
$$

7.3. Robin boundary conditions. We denote by $\Delta_{R, \Sigma}$ the self-adjoint extension corresponding to the orthogonal projector defined by $\Pi(\phi \oplus \varphi):=$ $\Pi_{\Sigma}^{\oplus}(\phi \oplus \varphi)=\left(\Pi_{\Sigma} \phi\right) \oplus\left(\Pi_{\Sigma} \varphi\right) \equiv R_{\Sigma}^{\oplus}(\phi \oplus \varphi):=(\phi \mid \Sigma) \oplus(\varphi \mid \Sigma)$ and to the self-adjoint operator $\Theta:=-\Theta_{R, \Sigma}$,

$$
\begin{aligned}
& \Theta_{R, \Sigma}: \operatorname{dom}\left(\Theta_{R, \Sigma}\right) \subseteq H_{\bar{\Sigma}}^{-\frac{3}{2}}(\Gamma) \oplus H_{\bar{\Sigma}}^{-\frac{1}{2}}(\Gamma) \rightarrow H^{\frac{3}{2}}(\Sigma) \oplus H^{\frac{1}{2}}(\Sigma), \\
& \Theta_{R, \Sigma}(\phi, \varphi)=\left(\left(\left(1 /[b]+\gamma_{0} S L\right) \phi+\left(\langle b\rangle /[b]+\hat{\gamma}_{0} D L\right) \varphi\right) \mid \Sigma\right) \\
& \oplus\left(\left(\left(\langle b\rangle /[b]+\hat{\gamma}_{1} S L\right) \phi+\left(b_{\mathrm{ex}} b_{\mathrm{in}} /[b]+\hat{\gamma}_{1} D L\right) \varphi\right) \mid \Sigma\right),
\end{aligned}
$$




$$
\begin{aligned}
& \operatorname{dom}\left(\Theta_{R, \Sigma}\right):=\left\{(\phi, \varphi) \in L_{\bar{\Sigma}}^{2}(\Gamma) \times H_{\frac{1}{\Sigma}}^{\frac{1}{2}}(\Gamma):\right. \\
& \left(\left(1 /[b]+\gamma_{0} S L\right) \phi+\left(\langle b\rangle /[b]+\hat{\gamma}_{0} D L\right) \varphi\right) \mid \Sigma \in H^{\frac{3}{2}}(\Sigma), \\
& \left.\left(\left(\langle b\rangle /[b]+\hat{\gamma}_{1} S L\right) \phi+\left(b_{\mathrm{ex}} b_{\mathrm{in}} /[b]+\hat{\gamma}_{1} D L\right) \varphi\right) \mid \Sigma \in H^{\frac{1}{2}}(\Sigma)\right\} .
\end{aligned}
$$

Here $b_{\text {in }}$ and $b_{\text {ex }}$ satisfy the same hypotheses as in subsection 6.3 and $b_{\text {in }}>$ $b_{\text {ex }}$. By Theorem 3.1 (see [29, Subsection 6.3]),

$$
\begin{aligned}
& \Delta_{R, \Sigma} u=\Delta u-\frac{4}{[b]}\left(\left(\langle b\rangle \gamma_{1} u-b_{\mathrm{ex}} b_{\text {in }} \gamma_{0} u\right) \delta_{\bar{\Sigma}}+\left(\gamma_{1} u-\langle b\rangle \gamma_{0} u\right) \nu \cdot \nabla \delta_{\bar{\Sigma}}\right), \\
& \operatorname{dom}\left(\Delta_{R, \Sigma}\right)=\left\{u \in H^{1}\left(\mathbb{R}^{n} \backslash \bar{\Sigma}\right) \cap H_{\Delta}^{0}\left(\mathbb{R}^{n} \backslash \Gamma\right):[\hat{\gamma}] u \in \operatorname{dom}\left(\Theta_{R, \Sigma}\right),\right. \\
&\left.\left(\gamma_{1}^{\text {in }} u-b_{\text {in }} \gamma_{0}^{\text {in }} u\right)\left|\Sigma=\left(\gamma_{1}^{\text {ex }} u-b_{\text {ex }} \gamma_{0}^{\text {ex }} u\right)\right| \Sigma=0\right\}
\end{aligned}
$$

is self-adjoint and

$$
\begin{aligned}
& \left(-\Delta_{R, \Sigma}+z\right)^{-1}-(-\Delta+z)^{-1} \\
= & -G_{z}\left(\Pi_{\Sigma}^{\oplus}\right)^{\prime}\left(R_{\Sigma}^{\oplus}\left[\begin{array}{cc}
1 /[b]+\gamma_{0} S L_{z} & \langle b\rangle /[b]+\gamma_{0} D L_{z} \\
\langle b\rangle /[b]+\gamma_{1} S L_{z} & b_{+} b_{-} /[b]+\gamma_{1} D L_{z}
\end{array}\right]\left(\Pi_{\Sigma}^{\oplus}\right)^{\prime}\right)^{-1} \\
& \times R_{\Sigma}^{\oplus} \gamma(-\Delta+z)^{-1}
\end{aligned}
$$

where $\left(\Pi_{\Sigma}^{\oplus}\right)^{\prime}$ is the orthogonal projection onto $H_{\bar{\Sigma}}^{-\frac{3}{2}}(\Gamma) \oplus H_{\bar{\Sigma}}^{-\frac{1}{2}}(\Gamma)$ and $G_{z}$ is defined in (2.6).

Remark 7.1. By [29, Remark 6.15], $\Delta_{R, \Sigma}$ depends only on $\Sigma, b_{\text {in }} \mid \Sigma$ and $b_{\text {ex }} \mid \Sigma$. Thus, by considering $\tilde{\Omega} \subset \mathbb{R}^{n}$ such that $\tilde{\Omega}_{\text {in }}=\tilde{\Omega} \subset \Omega_{\mathrm{ex}}, \Omega_{\mathrm{in}} \subset \tilde{\Omega}_{\mathrm{ex}}=$ $\mathbb{R}^{n} \backslash \tilde{\Omega}$ and $\Sigma \subset \tilde{\Gamma}=\partial \tilde{\Omega}$, it is possible to convert the assumption $b_{\text {in }}>b_{\text {ex }}$ into $b_{\text {ex }}>b_{\text {in }}$.

By Green's formula (2.8) and by Ehrling's lemma, for any $u \in \operatorname{dom}\left(\Delta_{R, \Sigma}\right) \subset$ $H^{1}\left(\mathbb{R}^{n} \backslash \Gamma\right) \cap H_{\Delta}^{0}\left(\mathbb{R}^{n} \backslash \Gamma\right)$ one has (here the Sobolev index $s$ belongs to $\left(\frac{1}{2}, 1\right)$ )

$$
\begin{aligned}
& \left\langle-\Delta_{R, \Sigma} u, u\right\rangle_{L^{2}\left(\mathbb{R}^{n}\right)} \\
= & \|\nabla u\|_{L^{2}\left(\Omega_{\mathrm{in}}\right)}^{2}+\|\nabla u\|_{L^{2}\left(\Omega_{\mathrm{ex}}\right)}^{2} \\
& -\left\langle b_{\mathrm{in}} \gamma_{0}^{\mathrm{in}} u_{\mathrm{in}}, \gamma_{0}^{\mathrm{in}} u_{\mathrm{in}}\right\rangle_{L^{2}(\Sigma)}+\left\langle b_{\mathrm{ex}} \gamma_{0}^{\mathrm{ex}} u_{\mathrm{ex}}, \gamma_{0}^{\mathrm{ex}} u_{\mathrm{ex}}\right\rangle_{L^{2}(\Sigma)} \\
\geq & \|\nabla u\|_{L^{2}\left(\Omega_{\mathrm{in}}\right)}^{2}+\|\nabla u\|_{L^{2}\left(\Omega_{\mathrm{ex}}\right)}^{2} \\
& -\left(\left\|b_{\mathrm{in}}\right\|_{L^{\infty}(\Gamma)}+\left\|b_{\mathrm{ex}}\right\|_{L^{\infty}(\Gamma)}\right)\left(\left\|\gamma_{0}^{\mathrm{in}} u_{\mathrm{in}}\right\|_{L^{2}(\Gamma)}^{2}+\left\|\gamma_{0}^{\mathrm{ex}} u_{\mathrm{ex}}\right\|_{L^{2}(\Gamma)}^{2}\right)
\end{aligned}
$$




$$
\begin{aligned}
& \geq\|\nabla u\|_{L^{2}\left(\Omega_{\mathrm{in}}\right)}^{2}+\|\nabla u\|_{L^{2}\left(\Omega_{\mathrm{ex}}\right)}^{2} \\
& \quad-c\left(\left\|b_{\mathrm{in}}\right\|_{L^{\infty}(\Gamma)}+\left\|b_{\mathrm{ex}}\right\|_{L^{\infty}(\Gamma)}\right)\left(\left\|u_{\mathrm{in}}\right\|_{H^{s}\left(\Omega_{\mathrm{in}}\right)}^{2}+\left\|u_{\mathrm{ex}}\right\|_{H^{s}\left(\Omega_{\mathrm{ex}}\right)}^{2}\right) \\
& \geq\|\nabla u\|_{L^{2}\left(\Omega_{\mathrm{in}}\right)}^{2}+\|\nabla u\|_{L^{2}\left(\Omega_{\mathrm{ex}}\right)}^{2} \\
& \quad-c\left(\left\|b_{\mathrm{in}}\right\|_{L^{\infty}(\Gamma)}+\left\|b_{\mathrm{ex}}\right\|_{L^{\infty}(\Gamma)}\right)\left(\epsilon\left(\left\|u_{\mathrm{in}}\right\|_{H^{1}\left(\Omega_{\mathrm{in}}\right)}^{2}+\left\|u_{\mathrm{ex}}\right\|_{H^{1}\left(\Omega_{\mathrm{in}}\right)}^{2}\right)+c_{\epsilon}\|u\|_{L^{2}\left(\mathbb{R}^{n}\right)}^{2}\right) \\
& \geq \\
& \geq \kappa_{\epsilon}\|u\|_{L^{2}\left(\mathbb{R}^{n}\right)}^{2}
\end{aligned}
$$

and so and so $\Delta_{\beta, \delta^{\prime}, \Sigma} \leq \kappa_{\epsilon}$. Then, by Theorems 5.6 and 3.7, one gets, for any $k>0$,

$$
\begin{aligned}
& s_{k}\left(\hat{\xi}, \hat{\xi}^{\prime}\right) \\
= & -\frac{i}{4 \pi}\left(\frac{k}{2 \pi}\right)^{n-2}\left\langle\left(R_{\Sigma}^{\oplus}\left[\begin{array}{cc}
1 /[b]+\gamma_{0} S L_{-k^{2}}^{+} & \langle b\rangle /[b]+\gamma_{0} D L_{-k^{2}}^{+} \\
\langle b\rangle /[b]+\gamma_{1} S L_{-k^{2}}^{+} & b_{+} b_{-} /[b]+\gamma_{1} D L_{-k^{2}}^{+}
\end{array}\right]\left(\Pi_{\Sigma}^{\oplus}\right)^{\prime}\right)^{-1}\right. \\
& \left.\times R_{\Sigma}^{\oplus} \gamma u_{k \xi^{\prime}}^{\circ}, R_{\Sigma}^{\oplus} \gamma u_{k \xi}^{\circ}\right\rangle .
\end{aligned}
$$

7.4. $\delta$-interactions. We denote by $\Delta_{\alpha, \delta, \Sigma}$ the self-adjoint extension corresponding to the orthogonal projector defined by $\Pi(\phi \oplus \varphi):=\left(\Pi_{\Sigma} \phi\right) \oplus 0 \equiv$ $(\phi \mid \Sigma) \oplus 0$ and to the self-adjoint operator $\Theta(\phi \oplus \varphi):=\left(-\Theta_{\alpha, D, \Sigma} \phi\right) \oplus 0$,

$$
\begin{gathered}
\Theta_{\alpha, D, \Sigma}: \operatorname{dom}\left(\Theta_{\alpha, D, \Sigma}\right) \subseteq H_{\bar{\Sigma}}^{-\frac{3}{2}}(\Gamma) \rightarrow H^{\frac{3}{2}}(\Sigma), \quad \Theta_{\alpha, D, \Sigma} \phi:=\left(\left(1 / \alpha+\gamma_{0} S L\right) \phi\right) \mid \Sigma, \\
\operatorname{dom}\left(\Theta_{\alpha, D, \Sigma}\right):=\left\{\phi \in L_{\bar{\Sigma}}(\Gamma):\left(\left(1 / \alpha+\gamma_{0} S L\right) \phi\right) \mid \Sigma \in H^{\frac{3}{2}}(\Sigma)\right\} .
\end{gathered}
$$

Here $\alpha$ satisfies the same hypothesis as in subsection 6.4 and we further require that it has constant sign on (each component of) $\Gamma$. By Theorem 3.1 (see [29, Subsection 6.4]; notice a misprint in [29, Corollary 6.21]: the condition $\left[\gamma_{0}\right] u=0$, implying $u \in H^{1}\left(\mathbb{R}^{n}\right)$, is missing in $\operatorname{dom}\left(\Delta_{\alpha, \delta, \Sigma}\right)$ ),

$$
\Delta_{\alpha, \delta, \Sigma} u=\Delta u-\alpha \gamma_{0} u \delta_{\bar{\Sigma}}
$$

$$
\begin{aligned}
& \operatorname{dom}\left(\Delta_{\alpha, \delta, \Sigma}\right) \\
= & \left\{u \in H^{1}\left(\mathbb{R}^{n}\right) \cap H^{2-}\left(\mathbb{R}^{n} \backslash \bar{\Sigma}\right) \cap H_{\Delta}^{0}\left(\mathbb{R}^{n} \backslash \Gamma\right):\left[\gamma_{1}\right] u \in \operatorname{dom}\left(\Theta_{\alpha, D, \Sigma}\right),\right. \\
& \left.\left(\alpha \gamma_{0} u-\left[\gamma_{1}\right] u\right) \mid \Sigma=0\right\}
\end{aligned}
$$

is self-adjoint and

$$
\begin{aligned}
& \left(-\Delta_{\alpha, \delta, \Sigma}+z\right)^{-1} u \\
= & (-\Delta+z)^{-1}-S L_{z} \Pi_{\Sigma}^{\prime}\left(R_{\Sigma}\left(1+\alpha \gamma_{0} S L_{z}\right) \Pi_{\Sigma}^{\prime}\right)^{-1} R_{\Sigma} \alpha \gamma_{0}(-\Delta+z)^{-1} .
\end{aligned}
$$


For any $u \in \operatorname{dom}\left(\Delta_{\alpha, \delta, \Sigma}\right)$, by Ehrling's lemma, one has (here the Sobolev index $s$ belongs to $\left.\left(\frac{1}{2}, 1\right)\right)$

$$
\begin{aligned}
\left\langle-\Delta_{\alpha, \delta, \Sigma} u, u\right\rangle_{L^{2}\left(\mathbb{R}^{n}\right)} & =\langle-\Delta u, u\rangle_{-1,1}+\left\langle\alpha \gamma_{0} u, \gamma_{0} u\right\rangle_{L^{2}(\Sigma)} \\
& =\|\nabla u\|_{L^{2}\left(\mathbb{R}^{n}\right)}^{2}+\left\langle\alpha \gamma_{0} u, \gamma_{0} u\right\rangle_{L^{2}(\Sigma)} \\
& \geq\|\nabla u\|_{L^{2}\left(\mathbb{R}^{n}\right)}^{2}-\|\alpha\|_{L^{\infty}(\Gamma)}\left\|\gamma_{0} u\right\|_{L^{2}(\Gamma)}^{2} \\
& \geq\|\nabla u\|_{L^{2}\left(\mathbb{R}^{n}\right)}^{2}-c\|\alpha\|_{L^{\infty}(\Gamma)}\|u\|_{H^{s}(\Omega)}^{2} \\
& \geq\|\nabla u\|_{L^{2}\left(\mathbb{R}^{n}\right)}^{2}-c\|\alpha\|_{L^{\infty}(\Gamma)}\left(\epsilon\|u\|_{H^{1}(\Omega)}^{2}+c_{\epsilon}\|u\|_{L^{2}(\Omega)}^{2}\right) \\
& \geq-\kappa_{\epsilon}\|u\|_{L^{2}(\Omega)}^{2}
\end{aligned}
$$

and so $\Delta_{\alpha, \delta, \Sigma} \leq \kappa_{\epsilon}$. By Theorems 5.6 and 3.7, one gets, for any $k>0$,

$s_{k}\left(\hat{\xi}, \hat{\xi}^{\prime}\right)=-\frac{i}{4 \pi}\left(\frac{k}{2 \pi}\right)^{n-2}\left\langle\left(R_{\Sigma}\left(1+\alpha \gamma_{0} S L_{z}\right) \Pi_{\Sigma}^{\prime}\right)^{-1} R_{\Sigma} \alpha \gamma_{0} u_{k \hat{\xi}^{\prime}}^{\circ}, R_{\Sigma} \gamma_{0} u_{k \hat{\xi}}^{\circ}\right\rangle$.

7.5. $\delta^{\prime}$-interaction. We denote by $\Delta_{\beta, \delta^{\prime}, \Sigma}$ the self-adjoint extension corresponding to the orthogonal projector defined by $\Pi(\phi \oplus \varphi):=0 \oplus\left(\Pi_{\Sigma} \varphi\right) \equiv$ $0 \oplus(\varphi \mid \Sigma)$ and to the self-adjoint operator $\Theta(\phi \oplus \varphi):=0 \oplus\left(-\Theta_{\beta, N, \Sigma}\right) \varphi$,

$$
\begin{gathered}
\Theta_{\beta, N, \Sigma}: \operatorname{dom}\left(\Theta_{\beta, N, \Sigma}\right) \subseteq H_{\bar{\Sigma}}^{-\frac{1}{2}}(\Gamma) \rightarrow H^{\frac{1}{2}}(\Sigma), \\
\Theta_{\beta, N, \Sigma} \phi:=\left(\left(-1 / \beta+\hat{\gamma}_{1} D L\right) \phi\right) \mid \Sigma, \\
\operatorname{dom}\left(\Theta_{\beta, N, \Sigma}\right):=\left\{\varphi \in H_{\frac{1}{\Sigma}}^{\frac{1}{2}}(\Gamma):\left(\left(-1 / \beta+\hat{\gamma}_{1} D L\right) \varphi\right) \mid \Sigma \in H^{\frac{1}{2}}(\Sigma)\right\} .
\end{gathered}
$$

Here $\beta$ satisfies the same hypothesis as in subsection 6.5. By Theorem 3.1 (see [29, Subsection 6.5]; notice a misprint in [29, Corollary 6.26]: the condition $\left[\hat{\gamma}_{1}\right] u=0$ is missing in $\left.\operatorname{dom}\left(\Delta_{\beta, \delta^{\prime}, \Sigma}\right)\right)$,

$$
\Delta_{\beta, \delta^{\prime}, \Sigma} u=\Delta u-\beta \gamma_{1} u \nu \cdot \nabla \delta_{\bar{\Sigma}},
$$

$$
\begin{aligned}
& \operatorname{dom}\left(\Delta_{\beta, \delta^{\prime}, \Sigma}\right) \\
= & \left\{u \in H^{1}\left(\mathbb{R}^{n} \backslash \bar{\Sigma}\right) \cap H_{\Delta}^{0}\left(\mathbb{R}^{n} \backslash \Gamma\right):\left[\gamma_{0}\right] u \in \operatorname{dom}\left(\Theta_{\beta, N, \Sigma}\right),\left[\hat{\gamma}_{1}\right] u=0,\right. \\
& \left.\left(\beta \hat{\gamma}_{1} u-\left[\gamma_{0}\right] u\right) \mid \Sigma=0\right\}
\end{aligned}
$$

is self-adjoint and its resolvent is given by

$$
\begin{aligned}
& \left(-\Delta_{\beta, \delta^{\prime}, \Sigma}+z\right)^{-1} u \\
= & (-\Delta+z)^{-1}+D L_{z} \Pi_{\Sigma}^{\prime}\left(R_{\Sigma}\left(1-\beta \hat{\gamma}_{1} D L_{z}\right) \Pi_{\Sigma}^{\prime}\right)^{-1} R_{\Sigma} \beta \gamma_{1}(-\Delta+z)^{-1} .
\end{aligned}
$$


By Green's formula (2.8) and by Ehrling's lemma, for any $u \in \operatorname{dom}\left(\Delta_{\beta, \delta^{\prime}, \Sigma}\right) \subset$ $H^{1}\left(\mathbb{R}^{n} \backslash \Gamma\right) \cap H_{\Delta}^{0}\left(\mathbb{R}^{n} \backslash \Gamma\right)$ one has (here the Sobolev index $s$ belongs to $\left(\frac{1}{2}, 1\right)$ )

$$
\begin{aligned}
& \left\langle-\Delta_{\beta, \delta^{\prime}, \Sigma} u, u\right\rangle_{L^{2}\left(\mathbb{R}^{n}\right)} \\
= & \|\nabla u\|_{L^{2}\left(\Omega_{\mathrm{in}}\right)}^{2}+\|\nabla u\|_{L^{2}\left(\Omega_{\mathrm{ex}}\right)}^{2}+\left\langle(1 / \beta)\left[\gamma_{0}\right] u,\left[\gamma_{0}\right] u\right\rangle_{L^{2}(\Sigma)} \\
\geq & \|\nabla u\|_{L^{2}\left(\Omega_{\mathrm{in}}\right)}^{2}+\|\nabla u\|_{L^{2}\left(\Omega_{\mathrm{ex}}\right)}^{2}-2\|1 / \beta\|_{L^{\infty}(\Gamma)}\left(\left\|\gamma_{0}^{\mathrm{in}} u_{\mathrm{in}}\right\|_{L^{2}(\Gamma)}^{2}+\left\|\gamma_{0}^{\mathrm{ex}} u_{\mathrm{ex}}\right\|_{L^{2}(\Gamma)}^{2}\right) \\
\geq & \|\nabla u\|_{L^{2}\left(\Omega_{\mathrm{in}}\right)}^{2}+\|\nabla u\|_{L^{2}\left(\Omega_{\mathrm{ex}}\right)}^{2}-c\|1 / \beta\|_{L^{\infty}(\Gamma)}\left(\left\|u_{\mathrm{in}}\right\|_{H^{s}\left(\Omega_{\mathrm{in}}\right)}^{2}+\left\|u_{\mathrm{ex}}\right\|_{H^{s}\left(\Omega_{\mathrm{ex}}\right)}^{2}\right) \\
\geq & \|\nabla u\|_{L^{2}\left(\Omega_{\mathrm{in}}\right)}^{2}+\|\nabla u\|_{L^{2}\left(\Omega_{\mathrm{ex}}\right)}^{2}-c\|1 / \beta\|_{L^{\infty}(\Gamma)}\left(\epsilon\left(\left\|u_{\mathrm{in}}\right\|_{H^{1}\left(\Omega_{\mathrm{in}}\right)}^{2}+\left\|u_{\mathrm{ex}}\right\|_{H^{1}\left(\Omega_{\mathrm{in}}\right)}^{2}\right)\right. \\
& \left.+c_{\epsilon}\|u\|_{L^{2}\left(\mathbb{R}^{n}\right)}^{2}\right) \geq-\kappa_{\epsilon}\|u\|_{L^{2}\left(\mathbb{R}^{n}\right)}^{2}
\end{aligned}
$$

and so and so $\Delta_{\beta, \delta^{\prime}, \Sigma} \leq \kappa_{\epsilon}$. Then, by Theorems [5.6] and 3.7, one gets, for any $k>0$,

$s_{k}\left(\hat{\xi}, \hat{\xi}^{\prime}\right)=-\frac{i}{4 \pi}\left(\frac{k}{2 \pi}\right)^{n-2}\left\langle\left(R_{\Sigma}\left(1-\beta \hat{\gamma}_{1} D L_{-k^{2}}^{+}\right) \Pi_{\Sigma}^{\prime}\right)^{-1} R_{\Sigma} \beta \gamma_{1} u_{k \hat{\xi}^{\prime}}^{\circ}, R_{\Sigma} \gamma_{1} u_{k \hat{\xi}}^{\circ}\right\rangle$.

\section{REFERENCES}

[1] V.M. Adamyan, B.S. Pavlov: Zero-radius potentials and M. G. Kreı̆n's formula for generalized resolvents. J. Soviet Math. 42 (1988), 1537-1550.

[2] S. Agmon: Spectral properties of Schrödinger operators and Scattering Theory. Ann. Scuola Sup. Pisa (IV) 11 (1975), 151-218.

[3] S. Albeverio, J. Brasche, V. Koshmanenko: Lippman-Schwinger Equation in the Singular Perturbation Theory. Methods Func. Anal. Topology 3 (1997), 1-27.

[4] S. Albeverio, P. Kurasov: Singular perturbations of differential operators. Cambridge University Press, Cambridge, 2000.

[5] P. Alsholm, G. Schmidt: Spectral and scattering theory for Schrödinger operators. Arch. Rational Mech. Anal. 40 (1970), 281-311.

[6] H. Baumgärtel, M. Wollenberg: Mathematical Scattering Theory, Akademie-Verlag, Berlin, 1983.

[7] M. Ben-Artzi, A. Devinatz: The limiting absorption principle for partial differential operators. Memoirs of the AMS vol. 364, 1987.

[8] J. Behrndt, M. Langer, V. Lotoreichik: Spectral Estimates for Resolvent Differences of Self-Adjoint Elliptic Operators. Integr. Equ. Oper. Theory 77 (2013), 1-37.

[9] J. Behrndt, M. Langer, V. Lotoreichik: Schrödinger operators with $\delta$ - and $\delta^{\prime}$ potentials supported on hypersurfaces. Ann. Henri Poincaré 14 (2013), 385-423.

[10] J. Behrndt, M.M. Malamud and H. Neidhardt: Scattering matrices and Weyl functions. Proc. London Math. Soc. 97 (2008), 568-598.

[11] J. Behrndt, M.M. Malamud and H. Neidhardt: Finite rank perturbations, scattering matrices and inverse problems. Operator Theory Advances Applications 198 (2009), 61-85.

[12] J. Behrndt, M.M. Malamud, H. Neidhardt: Scattering matrices and Dirichlet-toNeumann maps. Preprint 2015, arXiv:1511.02376.

[13] M.S. Birman: On the Self-Adjoint Extensions of Positive Definite Operators. Mat. Sbornik 38 (1956), 431-450 (in Russian). English translation by M. Khotyakov and A. Michelangeli available at http://urania.sissa.it/xmlui/handle/1963/34443 
[14] M.S. Birman: Perturbations of the continuous spectrum of a singular elliptic operator by varying the boundary and the boundary conditions. Vestn. Leningr. Univ. 17 (1962), 22-55 (in Russian). English translation in Amer. Math. Soc. Transl. (2) 225 (2008), 19-53.

[15] M.S. Birman, D.R. Yafaev: A general scheme in the stationary scattering theory. In M.S. Birman (editor), Problems in Mathematical Physics. Leningrad. Univ., Leningrad, 1987 (in Russian). English translation in Amer. Math. Soc. Trans. (2) 157 (1993), 87-112.

[16] J.F. Brasche, P. Exner, Yu. A. Kuperin: Schrödinger operators with singular interactions. J. Math. Anal. Appl. 184 (1994), 112-139.

[17] M. Dauge: Elliptic boundary value problems on corner domains. Lecture Notes in Mathematics vol. 1341. Springer, Berlin, 1988.

[18] R. Dautray, J-L. Lions: Mathematical Analysis and Numerical Methods for Science and Technology. Vol. 1: Physical Origins and Classical Methods. Springer, Berlin, 1990.

[19] J.-P. Eckmann, C.-A. Pillet: Spectral duality for planar billiards. Comm. Math. Phys. 170 (1995), 283-313.

[20] J.-P. Eckmann, C.-A. Pillet: Scattering phases and density of states for exterior domains. Ann. Inst. H. Poincaré Phys. Théor. 62 (1995), 383-399.

[21] J.-P. Eckmann, C.-A. Pillet: Zeta functions with Dirichlet and Neumann boundary conditions for exterior domains. Helv. Phys. Acta 70 (1997), 44-65.

[22] P. Exner, J. Rohleder: Generalized interactions supported on hypersurfaces. J. Math. Phys. 57 (2016), 041507.

[23] P. Grisvard: Singularities in boundary value problems. Masson, Paris, 1992.

[24] G. Grubb: A characterization of the non-local boundary value problems associated with an elliptic operator. Ann. Scuola Norm. Sup. Pisa (3) 22 (1968), 425-513.

[25] T. Ikebe: Eigenfunction expansions associated with the Schroedinger operators and their applications to scattering theory. Arch. Rational Mech. Anal. 5 (1960), 1-34.

[26] T. Ikebe, S. Shimada: Spectral and scattering theory for the Schrödinger operators with penetrable wall potentials. J. Math. Kyoto Univ. 31 (1991), 219-258.

[27] T. Kato: Perturbation theory for Linear Operators. Springer, 1976.

[28] M.G. Kreĭn: The Theory of Self-Adjoint Extensions of Half-Bounded Hermitean Operators and Their Applications. Mat. Sbornik 20(62) (1947), 431-459 (in Russian).

[29] A. Mantile. A. Posilicano, M. Sini: Self-adjoint elliptic operators with boundary conditions on not closed hypersurfaces. J. Differential Equations 261 (2016), 1-55.

[30] W. McLean: Strongly elliptic systems and boundary integral equations. Cambridge University Press, Cambrdige, 2000.

[31] R. Leis: Initial Boundary Value Problems in Mathematical Physics. B. G. Teubner, Stuttgart, 1986.

[32] J-L. Lions E. Magenes: Non-homogeneous Boundary Value Problems and Applications. Springer, New York, 1972.

[33] A. Posilicano: A Krĕın-like formula for singular perturbations of self-adjoint operators and applications. J. Funct. Anal. 183 (2001), 109-147.

[34] M. Reed and B. Simon: Methods of modern mathematical physics Vol. 4: Analysis of Operators. Academic Press. New York, 1978.

[35] W. Renger: Limiting absorption principle for singularly perturbed operators. Math. Nachr. 228 (2001), 163-187.

[36] W. Renger: Stability of limiting absorption principle under singular perturbations. In Mathematical results in quantum mechanics Prague, 1998, Oper. Theory Adv. Appl. 108, 351-357, Birkhäuser, Basel, 1999. 
LIMITING ABSORPTION PRINCIPLE, EIGENFUNCTIONS AND SCATTERING 39

[37] G. Schmidt: On the Representation of the Potential Scattering Operator in Quantum Mechanics. J. Differential Equations 7 (1970), 389-394.

[38] S. Shimada: Low energy scattering with a penetrable wall interaction. J. Math. Kyoto Univ. 34 (1994), 95-147.

[39] M.L. Višik: On General Boundary Problems for Elliptic Differential Equations. Trudy Mosc. Mat. Obsv. 1 (1952) 187-246 (in Russian); English translation in Amer. Math. Soc. Trans. (2) 24 (1963), 107-172.

[40] D. R. Yafaev. Mathematical Scattering Theory. General theory. American Mathematical Society, 1992.

[41] D. R. Yafaev. On the scattering matrix for perturbations of constant sign. Ann. Inst. H. Poincaré Phys. Théor. 57 (1992), 361-384.

Laboratoire de Mathématiques, Université de Reims - FR3399 CNRS, Moulin DE LA Housse BP 1039, 51687 Reims, France

DiSAT, Sezione di Matematica, Università dell'Insubria, Via Valleggio 11, I-22100 COMO, ITALY

RiCAm, Austrian Academy of Sciences, Altenbergerstr. 69, A-4040 Linz, Austria

E-mail address: andrea.mantile@univ-reims.fr

E-mail address: andrea.posilicano@uninsubria.it

E-mail address: mourad.sini@oeaw.ac.at 